\title{
One-dimensional kinetic description of nonlinear traveling-pulse and traveling-wave disturbances in long coasting charged particle beams
}

\author{
Ronald C. Davidson ${ }^{1}$ and Hong Qin ${ }^{1,2}$ \\ ${ }^{1}$ Plasma Physics Laboratory, Princeton University, Princeton, New Jersey 08543, USA \\ ${ }^{2}$ School of Nuclear Science and Technology and Department of Modern Physics, \\ University of Science and Technology of China, Hefei, Anhui 230026, China \\ (Received 15 April 2015; published 21 September 2015)
}

\begin{abstract}
This paper makes use of a one-dimensional kinetic model to investigate the nonlinear longitudinal dynamics of a long coasting beam propagating through a perfectly conducting circular pipe with radius $r_{w}$. The average axial electric field is expressed as $\left\langle E_{z}\right\rangle=-(\partial / \partial z)\langle\phi\rangle=-e_{b} g_{0} \partial \lambda_{b} / \partial z-e_{b} g_{2} r_{w}^{2} \partial^{3} \lambda_{b} / \partial z^{3}$, where $g_{0}$ and $g_{2}$ are constant geometric factors, $\lambda_{b}(z, t)=\int d p_{z} F_{b}\left(z, p_{z}, t\right)$ is the line density of beam particles, and $F_{b}\left(z, p_{z}, t\right)$ satisfies the 1D Vlasov equation. Detailed nonlinear properties of traveling-wave and traveling-pulse (soliton) solutions with time-stationary waveform are examined for a wide range of system parameters extending from moderate-amplitudes to large-amplitude modulations of the beam charge density. Two classes of solutions for the beam distribution function are considered, corresponding to: (i) the nonlinear waterbag distribution, where $F_{b}=$ const in a bounded region of $p_{z}$-space; and (ii) nonlinear Bernstein-Green-Kruskal (BGK)-like solutions, allowing for both trapped and untrapped particle distributions to interact with the self-generated electric field $\left\langle E_{z}\right\rangle$.
\end{abstract}

DOI: 10.1103/PhysRevSTAB.18.094201

PACS numbers: 29.27.Bd, 52.25.Dg

\section{INTRODUCTION}

High-energy accelerators and transport systems [1-6] have a wide variety of applications ranging from basic research in high energy and nuclear physics, to applications such as spallation neutron sources, medical physics, and heavy ion fusion. As a consequence, it is increasingly important to develop an improved understanding of collective processes and the nonlinear dynamics of intense charged particle beam systems. While there has been considerable progress in three-dimensional numerical and analytical investigations of the nonlinear VlasovMaxwell equations describing intense beam propagation, there is also considerable interest in the development and application of simplified one-dimensional kinetic models to describe the longitudinal nonlinear dynamics of long coasting beams [7-15] in linear (linac) or large-majorradius ring geometries. The present paper employs the onedimensional kinetic formalism recently developed by Davidson and Startsev [14] for a long coasting beam propagating through a perfecting conducting circular pipe with radius $r_{w}$. In Ref. [14] the average longitudinal electric field is expressed as $\left\langle E_{z}\right\rangle(z, t)=-(\partial / \partial z)\langle\phi\rangle(z, t)=$ $-e_{b} g_{0} \partial \lambda_{b} / \partial z-e_{b} g_{2} r_{w}^{2} \partial^{3} \lambda_{b} / \partial z^{3}$, where $e_{b}$ is the particle charge, $g_{0}$ and $g_{2}$ are constant geometric factors that depend on the location of the conducting wall and the shape of the

Published by the American Physical Society under the terms of the Creative Commons Attribution 3.0 License. Further distribution of this work must maintain attribution to the author(s) and the published article's title, journal citation, and DOI. transverse density profile, and $\lambda_{b}(z, t)=\int d p_{z} F_{b}\left(z, p_{z}, t\right)$ is the line density. In a previous application of the $1 \mathrm{D}$ kinetic formalism developed in Ref. [14], the analyses in Ref. [15] assumed that the longitudinal distribution $F_{b}\left(z, p_{z}, t\right)$ corresponded to a so-called waterbag distribution [16-19], where $F_{b}=$ const within moving boundaries in the phase space $\left(z, p_{z}\right)$. The weakly nonlinear analysis in Ref. [15] showed that disturbances moving near the sound speed evolve according to the Korteweg-deVries (KdV) equation [20-24]. The classical KdV equation, which arises in several areas of nonlinear physics in which there are cubic dispersive corrections to sound-wave-like signal propagation, also has the appealing feature that it is exactly solvable using inverse scattering techniques.

While the analysis in Ref. [15] reveals many interesting properties of the nonlinear evolution of longitudinal disturbances in intense charged particle beams, it is restricted to the weakly nonlinear regime. In the present analysis, we remove the restriction to the weakly nonlinear regime, and make use of the 1D kinetic model developed in Ref. [14], allowing for moderate to large-amplitude modulation in the charge density of the beam particles. The organization of this paper is the following. In Sec. II, the 1D kinetic model [14] is briefly reviewed (Sec. II A), and exact (local and nonlocal) nonlinear conservation constraints are derived (Sec. II B) for the conservation of particle number, momentum, and energy per unit length of the beam, making use of the nonlinear Vlasov equation for $F_{b}\left(z, p_{z}, t\right)$ in Eq. (1), and the expression for $\langle E\rangle(z, t)$ in Eq. (2). Removing the assumption of weak nonlinearity made in Ref. [15], Sec. III focuses on use of the fully nonlinear kinetic waterbag 
model (Sec. III A) to investigate detailed properties of nonlinear pulselike (soliton) or periodic traveling-wave disturbances propagating with constant normalized velocity $M=$ const relative to the beam frame (Sec. III B). In normalized variables, $Z^{\prime}=Z-M T$ and $T^{\prime}=T$, the waveform of the disturbance is assumed to be time-stationary $\left(\partial / \partial T^{\prime}=0\right)$ in the frame moving with velocity $M=$ const relative to the beam frame. Nonlinear solutions are examined over a wide range of system parameters, including regimes where the modulation in beam line density $\lambda_{b}$ exceeds $50 \%$, corresponding to a strongly bunched beam. Finally, in Sec. IV we examine the kinetic model based on Eqs. (9) and (10) [equivalent to Eqs. (1) and (2)] for an even broader class of distribution functions $F_{b}\left(z, p_{z}, t\right)$, recognizing that Eqs. (9) and (10) are Galilean invariant. [Keep in mind that the variables $\left(z, p_{z}, t\right)$ are in the beam frame, where the particle motion is assumed to be nonrelativistic.] Introducing the appropriately scaled variables (see Sec. IV) $Z^{\prime}=Z-M T, V_{z}^{\prime}=V_{z}-M, T^{\prime}=T$, where $M=$ const, we transform Eqs. (9) and (10) to primed variables, and look for solutions that are time stationary $\left(\partial / \partial T^{\prime}=0\right)$ in the frame moving with velocity $M=$ const relative to the beam frame. The analysis in Sec. IV parallels the original Bernstein-Greene-Kruskal (BGK) formulation of BGK solutions to the 1D Vlasov-Poisson equations [25,26], except for the fact that Eq. (10), which connects the effective potential $\langle\phi\rangle(z, t)$ to the line density $\lambda_{b}(z, t)$, has a very different structure than the 1D Poisson's equation used in the original BGK analysis. Depending on the choices of trapped-particle and untrapped-particle distribution functions, the kinetic model described in Sec. IV supports a broad range of nonlinear pulselike (soliton) solutions and periodic traveling-wave solutions that have time-stationary waveform in the frame moving with velocity $M=$ const relative to the beam frame. Similar to Sec. III B, the modulation of beam line density can have large amplitude, corresponding to a strong bunching of the beam particles. Specific examples are presented in Sec. IV corresponding to nonlinear periodic traveling-wave solutions.

\section{THEORETICAL MODEL AND ASSUMPTIONS}

This section provides a brief summary of the onedimensional kinetic g-factor model (Sec. II A) developed by Davidson and Startsev [14] to describe the nonlinear longitudinal dynamics of a long coasting beam propagating in the $z$-direction through a circular, perfectly conducting pipe with radius $r_{w}$. The 1D kinetic Vlasov equation for the distribution function $F_{b}\left(z, p_{z}, t\right)$ is used (Sec. II B) to derive several important conservation laws (both local and global) corresponding to conservation of particle number, momentum, and energy per unit length of the charge bunch. The results in Secs. II A and II B form the basis for the nonlinear traveling-wave and traveling-pulse solutions studied in Secs. III and IV.

\section{A. Theoretical model and assumptions}

This paper makes use of a one-dimensional kinetic model [14] that describes the nonlinear dynamics of the longitudinal distribution function $F_{b}\left(z, p_{z}, t\right)$, the average self-generated axial electric field $\left\langle E_{z}\right\rangle(z, t)$, and the line density $\lambda_{b}(z, t)=\int d p_{z} F_{b}\left(z, p_{z}, t\right)$, for an intense charged particle beam propagating in the $z$-direction through a circular, perfectly conducting pipe with radius $r_{w}$. For simplicity, the analysis is carried out in the beam frame, where the longitudinal particle motion in $\left(z, p_{z}\right)$ phase space is assumed to be nonrelativistic, and the beam intensity is assumed to be sufficiently low that the beam edge radius $r_{b}$ and rms radius $R_{b}=\left\langle r^{2}\right\rangle^{1 / 2}=\left\langle x^{2}+y^{2}\right\rangle^{1 / 2}$ have a negligibly small dependence on line density $\lambda_{b}$. Furthermore, properties such as the number density $n_{b}(r, z, t)$ of beam particles are assumed to be azimuthally symmetric about the beam axis $(\partial / \partial \theta=0)$, where $x=$ $r \cos \theta$ and $y=r \sin \theta$ are cylindrical polar coordinates. Finally, the axial spatial variation in the line density $\lambda_{b}(z, t)=2 \pi \int_{0}^{r_{w}} d r n_{b}(r, z, t)$ is assumed to be sufficiently slow that $k_{z}^{2} r_{w}^{2} \ll 1$, where $\partial / \partial z \backsim k_{z} \backsim L_{z}^{-1}$ is the inverse length scale of the z-variation of beam properties.

Making use of these assumptions, it can be shown that the one-dimensional kinetic equation describing the nonlinear evolution of the longitudinal distribution function $F_{b}\left(z, p_{z}, t\right)$ and average longitudinal electric field $\left\langle E_{z}\right\rangle(z, t)$ can be expressed in the beam frame correct to order $k_{z}^{2} r_{w}^{2}$ as [14]

$$
\frac{\partial}{\partial t} F_{b}+v_{z} \frac{\partial}{\partial z} F_{b}+e_{b}\left\langle E_{z}\right\rangle \frac{\partial}{\partial p_{z}} F_{b}=0,
$$

and

$$
\frac{e_{b}}{m_{b}}\left\langle E_{z}\right\rangle=-\frac{U_{b 0}^{2}}{\lambda_{b 0}} \frac{\partial}{\partial z} \lambda_{b}-\frac{r_{w}^{2} U_{b 2}^{2}}{\lambda_{b 0}} \frac{\partial^{3} \lambda_{b}}{\partial z^{3}} .
$$

Here, $e_{b}$ and $m_{b}$ are the charge and rest mass of a beam particle, and $\lambda_{b 0}=$ const is a measure of the characteristic line density of beam particles, e.g., its average value. Moreover, the constants $U_{b 0}^{2}$ and $U_{b 2}^{2}$ have dimensions of speed-squared, and are defined by

$$
U_{b 0}^{2}=\frac{\lambda_{b 0} g_{0} e_{b}^{2}}{m_{b}}, \quad U_{b 2}^{2}=\frac{\lambda_{b 0} g_{2} e_{b}^{2}}{m_{b}}
$$

where $g_{0}$ and $g_{2}$ are the geometric factors defined by [14]

$$
\begin{gathered}
g_{0}=2 \int_{0}^{r_{w}} \frac{d r}{r}\left(2 \pi \int_{0}^{r} d r r \frac{n_{b}}{\lambda_{b}}\right)^{2}, \\
g_{2}=\frac{2}{r_{w}^{2}} \int_{0}^{r_{w}} \frac{d r}{r} 2 \pi\left(\int_{0}^{r} d r r \frac{n_{b}}{\lambda_{b}}\right) \\
\times \int_{0}^{r} d r r \int_{r}^{r_{w}} \frac{d r}{r}\left(2 \pi \int_{0}^{r} d r r \frac{n_{b}}{\lambda_{b}}\right) .
\end{gathered}
$$


In obtaining Eqs. (1)-(5), a perfectly conducting cylindrical wall with $E_{z}\left(r=r_{w}, z, t\right)=0$ has been assumed.

For the purposes of illustration, we consider the class of axisymmetric density profiles $n_{b}(r, z, t)$ of the form

$$
n_{b}= \begin{cases}\frac{\lambda_{b}}{\pi r_{b}^{2}} f\left(\frac{r}{r_{b}}\right), & 0 \leq r<r_{b}, \\ 0, & r_{b}<r \leq r_{w} .\end{cases}
$$

Here, $\lambda_{b}=\int d p_{z} F_{b}\left(z, p_{z}, t\right)=2 \pi \int_{0}^{r_{w}} d r n_{b}(r, z, t)$ is the line density, $r_{b}$ is the beam edge radius, assumed independent of $\lambda_{b}$, and $f\left(r / r_{b}\right)$ is the profile shape function with normalization $\int_{0}^{1} d x x f(x)=1 / 2$. As an example, for $f\left(r / r_{b}\right)=(n+1)\left(1-r^{2} / r_{b}^{2}\right)^{n}, n=0,1,2, \ldots$, over the interval $0 \leq r<r_{b}$, it can be shown that [14]

$$
\begin{aligned}
g_{0}= & \ln \left(\frac{r_{w}^{2}}{r_{b}^{2}}\right)+\sum_{m=1}^{n+1} \frac{n+1}{m(m+n+1)}, \\
g_{2}= & \frac{1}{2}\left[1-\frac{1}{(n+2)} \frac{r_{b}^{2}}{r_{w}^{2}}\left(1+\ln \frac{r_{w}^{2}}{r_{b^{2}}}\right)\right. \\
& \left.-\sum_{m=1}^{n+1} \frac{1}{m(m+n+2)} \frac{r_{b}^{2}}{r_{w}^{2}}\right] .
\end{aligned}
$$

From Eqs. (6)-(8), we note that $n=0$ corresponds to a step-function density profile; $n=1$ corresponds to a parabolic density profile; $n \geq 2$ corresponds to an even more peaked density profile; and that the precise values of $g_{0}$ and $g_{2}$ depend on the profile shape [14]. Finally, for the choice of shape function $f\left(r / r_{b}\right)=(n+1)\left(1-r^{2} / r_{b}^{2}\right)^{n}$, $n=0,1,2, \ldots$, it is readily shown that the mean-square beam radius is $R_{b}^{2}=\lambda_{b}^{-1} 2 \pi \int_{0}^{r_{w}} d r r r^{2} n_{b}=(n+2)^{-1} r_{b}^{2}$, so that for fixed value of rms radius $R_{b}$, the edge radius $r_{b}$ increases with increasing value of the density profile shape factor $n$.

\section{B. Conservation relations}

Equations (1) and (2) possess several important conservation laws, both local and global, corresponding to conservation of particle number, momentum, and energy per unit length. For present purposes we express $\left\langle E_{z}\right\rangle(z, t)=$ $-(\partial / \partial z)\langle\phi\rangle(z, t)$. Equations (1) and (2) then describe the evolution of $F_{b}\left(z, p_{z}, t\right)$ and $\langle\phi\rangle(z, t)$ according to

$$
\frac{\partial}{\partial t} F_{b}+v_{z} \frac{\partial}{\partial z} F_{b}-e_{b} \frac{\partial\langle\phi\rangle}{\partial z} \frac{\partial F_{b}}{\partial p_{z}}=0,
$$

where $v_{z}=p_{z} / m_{b}$ and

$$
e_{b} \frac{\partial}{\partial z}\langle\phi\rangle=m_{b} U_{b 0}^{2} \frac{\partial}{\partial z} N_{b}+m_{b} U_{b 2}^{2} r_{w}^{2} \frac{\partial^{3}}{\partial z^{3}} N_{b} .
$$

Here,

$$
N_{b}(z, t)=\frac{\lambda_{b}(z, t)}{\lambda_{b 0}}=\lambda_{b 0}^{-1} \int d p_{z} F_{b}\left(z, p_{z}, t\right)
$$

is a dimensionless measure of the line density $\lambda_{b}(z, t)$, and $\lambda_{b 0}=$ const is the characteristic (e.g., average) value of line density.

It is convenient to introduce the macroscopic moments

$$
\begin{gathered}
N_{b} V_{b}=N_{b}\left\langle v_{z}\right\rangle=\lambda_{b 0}^{-1} \int d p_{z} v_{z} F_{b}, \\
N_{b}\left\langle v_{z}^{n}\right\rangle=\lambda_{b 0}^{-1} \int d p_{z} v_{z}^{n} F_{b},
\end{gathered}
$$

where $N_{b}=\lambda_{b} / \lambda_{b 0}$ is defined in Eq. (11), and $V_{b}(z, t)=$ $\left(\int d p_{z} v_{z} F_{b}\right) /\left(\int d p_{z} F_{b}\right)$ is the average axial flow velocity in the beam frame. Note that the effective "pressure" $P_{b}(z, t)$ and "heat flow" $Q_{b}(z, t)$ are defined (relative to the average flow velocity $V_{b}$ ) by

$$
\begin{aligned}
P_{b}(z, t) & =\lambda_{b 0} N_{b} m_{b}\left\langle\left(v_{z}-V_{b}\right)^{2}\right\rangle \\
& =m_{b} \int d p_{z}\left(v_{z}-V_{b}\right)^{2} F_{b},
\end{aligned}
$$

and

$$
\begin{aligned}
Q_{b}(z, t) & =\lambda_{b 0} N_{b} m_{b}\left\langle\left(v_{z}-V_{b}\right)^{3}\right\rangle \\
& =m_{b} \int d p_{z}\left(v_{z}-V_{b}\right)^{3} F_{b},
\end{aligned}
$$

where $V_{b}(z, t)$ is the average flow velocity defined in Eq. (12).

We now make use of Eqs. (9) and (10) to derive the local and global conservation laws corresponding to the conservation of particle number, momentum, and energy per unit length of the beam. The subsequent analysis applies to the two classes of beam systems: (i) a very long, finitelength charge bunch $\left(L_{b} \gg r_{w}\right)$ with $N_{b}(z \rightarrow \pm \infty, t)=0$; and (ii) a circulating beam in a large-aspect-ratio $\left(R_{0} \gg r_{w}\right)$ ring with periodic boundary condition $N_{b}\left(z+2 \pi R_{0}, t\right)=$ $N_{b}(z, t)$ as the beam circulates around the ring with major radius $R_{0}$. (Here, $z$ can be viewed as the arc length around the perimeter of the ring with large radius $R_{0}$.)

\section{Number conservation}

From Eqs. (9), (11), and (12), operating on Eq. (9) with $\lambda_{b 0}^{-1} \int d p_{z} \cdots$, and integrating by parts with respect to $p_{z}$, we obtain

$$
\frac{\partial}{\partial t} N_{b}+\frac{\partial}{\partial z}\left(N_{b} V_{b}\right)=0
$$

where $N_{b}(z, t)=\lambda_{b}(z, t) / \lambda_{b 0}$ is the normalized line density, and $V_{b}(z, t)$ is the axial flow velocity [Eqs. (11) and (12)]. Equation (16) is a statement of local number conservation, i.e., the time rate of change of the local density, $\partial N_{b} / \partial t$, is equal to minus the derivative of the local flux of particles, $-(\partial / \partial z)\left(N_{b} V_{b}\right)$. If we integrate 
Eq. (16) over $z$, applying the boundary conditions described earlier in this section, we obtain

$$
\frac{\partial}{\partial t} \int d z N_{b}=0
$$

which corresponds to the global conservation of the number of beam particles.

\section{Momentum conservation}

We now operate on Eq. (9) with $\lambda_{b 0}^{-1} \int d p_{z} p_{z} \cdots$, where $p_{z}=m_{b} v_{z}$, and make use of Eqs. (12) and (13). This gives

$$
\frac{\partial}{\partial t} N_{b} m_{b} V_{b}+\frac{\partial}{\partial z} N_{b} m_{b}\left\langle v_{z} v_{z}\right\rangle+e_{b} N_{b} \frac{\partial}{\partial z}\langle\phi\rangle=0,
$$

where $-(\partial / \partial z)\langle\phi\rangle$ is defined in Eq. (10), and we have integrated by parts with respect to $p_{z}$ to obtain Eq. (18) from Eq. (9). Equation (18) can be expressed in an alternate form by making use of Eq. (10) to eliminate $e_{b}(\partial / \partial z)\langle\phi\rangle$ and combine Eqs. (12)-(14) to express

$$
N_{b} m_{b}\left\langle v_{z} v_{z}\right\rangle=N_{b} m_{b} V_{b} V_{b}+\lambda_{b 0}^{-1} P_{b}
$$

where $V_{b}(z, t)$ is the average flow velocity, and $P_{b}(z, t)$ is the effective pressure of the beam particles. Substituting Eqs. (10) and (19) into Eq. (18), we obtain

$$
\begin{aligned}
& \frac{\partial}{\partial t} N_{b} m_{b} V_{b}+\frac{\partial}{\partial z}\left\{N_{b} m_{b} V_{b} V_{b}+\lambda_{b 0}^{-1} P_{b}\right\} \\
& \quad+N_{b} m_{b}\left\{U_{b 0}^{2} \frac{\partial N_{b}}{\partial z}+U_{b 2}^{2} r_{w}^{2} \frac{\partial^{3} N_{b}}{\partial z^{3}}\right\} \\
& =\frac{\partial}{\partial t} N_{b} m_{b} V_{b}+\frac{\partial}{\partial z}\left\{N m_{b} V_{b} V_{b}+\lambda_{b 0}^{-1} P_{b}+\frac{1}{2} m_{b} U_{b 0}^{2} N_{b}^{2}\right\} \\
& \quad+\frac{\partial}{\partial z}\left\{m_{b} U_{b 2}^{2} r_{w}^{2}\left[N_{b} \frac{\partial^{2} N_{b}}{\partial z^{2}}-\frac{1}{2}\left(\frac{\partial N_{b}}{\partial z}\right)^{2}\right]\right\}=0
\end{aligned}
$$

Note that Eq. (20) expresses the local force balance equation in the form of a local conservation relation for the momentum density of a beam fluid element. Moreover, integrating Eq. (20) over $z$ and applying the boundary conditions described earlier in Sec. II gives

$$
\frac{\partial}{\partial t} \int d z N_{b} m_{b} V_{b}=0
$$

which corresponds to global momentum conservation.

\section{Energy conservation}

We now operate on Eq. (9) with $\lambda_{b 0}^{-1} \int d p_{z} \frac{1}{2} m_{b} v_{z}^{2} \cdots$ and make use of Eqs. (11)-(13) and $p_{z}=m_{b} v_{z}$. Integrating by parts with respect to $p_{z}$, we readily obtain

$$
\begin{aligned}
& \frac{\partial}{\partial z}\left(\frac{1}{2} N_{b} m_{b}\left\langle v_{z}^{2}\right\rangle\right)+\frac{\partial}{\partial z}\left(\frac{1}{2} N_{b} m_{b}\left\langle v_{z}^{3}\right\rangle\right)+e_{b} \frac{\partial\langle\phi\rangle}{\partial z} N_{b} V_{b} \\
& \quad=0
\end{aligned}
$$

From Eqs. (10) and (16), some straightforward algebraic manipulation gives

$$
\begin{aligned}
e_{b} \frac{\partial\langle\phi\rangle}{\partial z} N_{b} V_{b}= & \frac{\partial}{\partial z}\left[\frac{1}{2} m_{b} U_{b 0}^{2} N_{b}^{2}-\frac{1}{2} m_{b} U_{b 2}^{2} r_{w}^{2}\left(\frac{\partial N_{b}}{\partial z}\right)^{2}\right] \\
& +\frac{\partial}{\partial z}\left\{m_{b} U_{b 0}^{2}\left(N_{b} N_{b} V_{b}\right)+m_{b} U_{b 2}^{2} r_{w}^{2}\right. \\
& \left.\times\left[N_{b} V_{b} \frac{\partial^{2} N_{b}}{\partial z^{2}}+\frac{\partial N_{b}}{\partial z} \frac{\partial N_{b}}{\partial t}\right]\right\}
\end{aligned}
$$

Substituting Eq. (23) into Eq. (22) and rearranging terms, we obtain

$$
\begin{aligned}
\frac{\partial}{\partial t} & \left\{\frac{1}{2} N_{b} m_{b}\left\langle v_{z}^{2}\right\rangle+\frac{1}{2} m_{b} U_{b 0}^{2} N_{b}^{2}-\frac{1}{2} m_{b} U_{b 2}^{2} r_{w}^{2}\left(\frac{\partial N_{b}}{\partial z}\right)^{2}\right\} \\
& +\frac{\partial}{\partial z}\left\{\frac{1}{2} N_{b} m_{b}\left\langle v_{z}^{3}\right\rangle+m_{b} U_{b 0}^{2} N_{b}^{2} V_{b}+m_{b} U_{b 2}^{2} r_{w}^{2}\right. \\
& \left.\times\left[N_{b} V_{b} \frac{\partial^{2} N_{b}}{\partial z^{2}}+\frac{\partial N_{b}}{\partial z} \frac{\partial N_{b}}{\partial t}\right]\right\}=0
\end{aligned}
$$

which corresponds to local conservation of energy. Global energy conservation follows upon integrating Eq. (24) over $z$, which gives

$$
\begin{aligned}
& \frac{\partial}{\partial t} \int d z\left\{\frac{1}{2} N_{b} m_{b}\left\langle v_{z}^{2}\right\rangle+\frac{1}{2} m_{b} U_{b 0}^{2} N_{b}^{2}-\frac{1}{2} m_{b} U_{b 2}^{2} r_{w}^{2}\left(\frac{\partial N_{b}}{\partial z}\right)^{2}\right\} \\
& =0 .
\end{aligned}
$$

Note that Eq. (25) describes the balance in energy exchange between particle kinetic energy and electrostatic field energy. Moreover, the final two terms inside curly brackets in Eq. (25) correspond to electrostatic field energy, and the term inside the curly brackets proportional to $U_{b 0}^{2}$ is positive, whereas the term proportional to $U_{b 2}^{2}$ is manifestly negative. Because of the negative sign of the third term in Eq. (25), note that any increase in $\left(\partial N_{b} / \partial z\right)^{2}$ averaged over $z$ must compensated by a corresponding increase in the first two terms in Eq. (25).

To summarize, the local conservation laws in Eqs. (16), (18), and (24), and the global conservation laws in Eqs. (17), (21), and (25), provide powerful nonlinear constrains on the evolution of the normalized line density $N_{b}$, momentum density $N_{b} m_{b} V_{b}$, and kinetic energy density $N_{b} m_{b}\left\langle v_{z}^{2}\right\rangle /$ $2=N_{b} m_{b} V_{b}^{2} / 2+\lambda_{b 0}^{-1} P_{b} / 2$. Furthermore, these conservation constraints are exact consequences of the 1D nonlinear Vlasov equation (9) for $F_{b}\left(z, p_{z}, t\right)$, where $e_{b}(\partial / \partial z)\langle\phi\rangle$ $(z, t)$ is defined in Eq. (10), and $U_{b 0}^{2}$ and $U_{b 2}^{2}$ are expressed in terms of the geometric factors $g_{0}$ and $g_{2}$ in Eq. (3). 
Finally, the energy balance equation (22), the momentum balance equation (18), and the continuity equation (16) can be combined to give a dynamical equation for the evolution of the effective pressure $P_{b}(z, t)$ of the beam particles. We make use of $N_{b} m_{b}\left\langle v_{z}^{2}\right\rangle=N_{b} m_{b} V_{b}^{2}+\lambda_{b 0}^{-1} P_{b}$, to express

$$
\begin{aligned}
N_{b} m_{b}\left\langle v_{z}^{3}\right\rangle= & N_{b} m_{b}\left\langle\left(v_{z}-V_{b}+V_{b}\right)^{3}\right\rangle \\
= & N_{b} m_{b} V_{b}^{3}+3 N_{b} m_{b} V_{b}\left\langle\left(v_{z}-V_{b}\right)^{2}\right\rangle \\
& +N_{b} m_{b}\left\langle\left(v_{z}-V_{b}\right)^{3}\right\rangle \\
= & N_{b} m_{b} V_{b}^{3}+3 N_{b} V_{b} \lambda_{b 0}^{-1} P_{b}+\lambda_{b 0}^{-1} Q_{b},
\end{aligned}
$$

where $Q_{b}$ is the effective heat flow defined in Eq. (15). Without presenting algebraic details, some straightforward manipulation of Eq. (22) that makes use of Eqs. (16) and (18) then gives

$$
\left(\frac{\partial}{\partial t}+V_{b} \frac{\partial}{\partial z}\right) P_{b}+3 P_{b} \frac{\partial V_{b}}{\partial z}+\frac{\partial}{\partial z} Q_{b}=0 .
$$

To summarize, Eqs. (16), (20), and (27) describe the selfconsistent nonlinear evolution of $N_{b}(z, t), V_{b}(z, t)$ and $P_{b}(z, t)$. In the special case where the heat flow contribution $(\partial / \partial z) Q_{b}$ is negligibly small in Eq. (27), the pressure $P_{b}(z, t)$ evolves approximately according to

$$
\left(\frac{\partial}{\partial t}+V_{b} \frac{\partial}{\partial z}\right) P_{b}+3 P_{b} \frac{\partial V_{b}}{\partial z}=0
$$

The continuity equation (16) can be expressed as

$$
\left(\frac{\partial}{\partial t}+V_{b} \frac{\partial}{\partial z}\right) N_{b}+N_{b} \frac{\partial V_{b}}{\partial z}=0 .
$$

Combining Eqs. (27) and (28), we obtain

$$
\left(\frac{\partial}{\partial t}+V_{b} \frac{\partial}{\partial z}\right)\left(\frac{P_{b}}{N_{b}^{3}}\right)=0
$$

which can be integrated to give the triple-adiabatic pressure relation $\left(P_{b} / N_{b}^{3}\right)=$ const. Therefore, for negligibly small heat flow in Eq. (27), the macroscopic fluid model obtained by taking moments of the 1D Vlasov equation (9) closes, and the nonlinear evolution of $N_{b}, V_{b}$ and $P_{b}$ is described by Eqs. (16), (20), and (30).

In Sec. III, we discuss a particular choice of distribution function $F_{b}\left(z, p_{z}, t\right)$, corresponding to the so-called waterbag distribution, for which the heat flow $Q_{b}(z, t)$ is exactly zero during the nonlinear evolution of the system. In this case, the closure is exact, and the nonlinear evolution of the system is fully described by Eqs. (16), (20), and (30).

\section{COHERENT NONLINEAR STRUCTURES OBTAINED FROM THE KINETIC WATERBAG MODEL}

The 1D kinetic g-factor model based on Eqs. (1) and (2) can be used to determine the nonlinear evolution of the beam distribution function $F_{b}\left(z, p_{z}, t\right)$ for a broad range of system parameters and initial distribution functions. In this section, we examine Eqs. (1) and (2) for the class of exact solutions for $F_{b}\left(z, p_{z}, t\right)$ corresponding to the so-called waterbag distribution in which $F_{b}\left(z, p_{z}, t\right)$ has uniform density in phase space (Sec. III A). The subclass of coherent nonlinear traveling-wave and traveling-pulse solutions with undistorted waveform are then examined (Sec. III B) for disturbances traveling in the longitudinal direction with constant normalized velocity $M=$ const.

\section{A. Kinetic Waterbag model}

Equations (1) and (2), or equivalently, Eqs. (9) and (10) constitute the starting point in the present 1D kinetic description of the longitudinal nonlinear dynamics of a long coasting beam. The detailed wave excitations associated with Eqs. (9) and (10) of course depend on the form of the distribution function $F_{b}\left(z, p_{z}, t\right)$. For small-amplitude perturbations, Eqs. (1) and (2) support solutions corresponding to sound-wave-like disturbances with signal speed depending on $U_{b 0}$ and the momentum spread of $F_{b}$, and cubic dispersive modifications depending on $U_{b 2}$ [14].

In this section, we specialize to the class of exact nonlinear solutions for $F_{b}\left(z, p_{z}, t\right)$ to Eq. (1) corresponding to the waterbag distribution [15-19]

$F_{b}\left(z, p_{z}, t\right)= \begin{cases}A=\mathrm{const}, & m V_{b}^{-}(z, t)<p_{z}<m_{b} V_{b}^{+}(z, t), \\ 0, & \text { otherwise, }\end{cases}$

for $-\infty<z<\infty$ (long coasting beam in linear geometry) or $0<z<2 \pi R_{0}$ (large-aspect-ratio ring with major radius $R_{0}$ ). In Eq. (31), the distribution function $F_{b}=A$ remains constant within the boundary curves $m_{b} V_{b}^{-}$and $m_{b} V_{b}^{+}$, and zero outside. The boundary curves, $m_{b} V_{b}^{-}(z, t)$ and $m_{b} V_{b}^{+}(z, t)$, are assumed to be single-valued, and of course the boundary curves distort nonlinearly as the system evolves according to Eqs. (1) and (2) [or equivalently, Eqs. (9) and (10)]. We integrate across the two boundary curves in Eq. (31) by operating on Eq. (1) with

$$
\begin{aligned}
& \lim _{\epsilon \rightarrow 0^{+}} \int_{m_{b} V_{b}^{-}(1-\epsilon)}^{m_{b} V_{b}^{-}(1+\epsilon)} d p_{z} p_{z} \cdots, \quad \text { and } \\
& \lim _{\epsilon \rightarrow 0^{+}} \int_{m_{b} V_{b}^{+}(1-\epsilon)}^{m_{b} V_{b}^{+}(1+\epsilon)} d p_{z} p_{z} \cdots,
\end{aligned}
$$

where $p_{z}=m_{b} v_{z}$. Integrating by parts with respect to $p_{z}$, and taking the limit $\epsilon \rightarrow 0^{+}$, we obtain for the nonlinear evolution of the boundary curves $V_{b}^{-}(z, t)$ and $V_{b}^{+}(z, t)$ 


$$
\begin{aligned}
& \frac{\partial}{\partial t} V_{b}^{-}+V_{b}^{-} \frac{\partial}{\partial z} V_{b}^{-}=\frac{e_{b}}{m_{b}}\left\langle E_{z}\right\rangle, \\
& \frac{\partial}{\partial t} V_{b}^{+}+V_{b}^{+} \frac{\partial}{\partial z} V_{b}^{+}=\frac{e_{b}}{m_{b}}\left\langle E_{z}\right\rangle,
\end{aligned}
$$

where $\left\langle E_{z}\right\rangle$ is defined in Eq. (2).

To summarize, for each value of $z$, the waterbag distribution in Eq. (31) has a flat-top distribution in $p_{z}$ (also called a "top-hat" distribution) between the boundary curves $p_{z}=m_{b} V_{b}^{-}(z, t)$ and $p_{z}=m_{b} V_{b}^{+}(z, t)$. While the momentum derivative proportional to $\partial F_{b} / \partial p_{z}$ becomes singular at the boundaries, $p_{z}=m_{b} V_{b}^{-}(z, t)$ and $p_{z}=$ $m_{b} V_{b}^{+}(z, t)$, for the choice of waterbag distribution in Eq. (31), it is precisely this delta-function singularity that permits the integration in Eq. (32) over the Vlasov equation (1) to be carried out exactly, leading to the dynamical equations (33) and (34) for the boundary curves $m_{b} V_{b}^{-}(z, t)$ and $m_{b} V_{b}^{+}(z, t)$. Indeed, for the choice of waterbag distribution in Eq. (31), the nonlinear Vlasov equation (1) is exactly equivalent to the two dynamical equations (33) and (34) for $V_{b}^{-}(z, t)$ and $V_{b}^{+}(z, t)$. Furthermore, it can be shown exactly that if $F_{b}\left(z, p_{z}, t\right)$ has the form of the waterbag distribution in Eq. (31) at $t=0$, then the flat-top waterbag form is maintained at all subsequent times $t$ by the Vlasov equation (1), where the boundary curves $m_{b} V_{b}^{-}(z, t)$ and $m_{b} V_{b}^{+}(z, t)$ evolve nonlinearly according to Eqs. (33) and (34), and $\left\langle E_{z}\right\rangle$ is determined from Eq. (2).

The positive features of the choice of waterbag distribution function in Eq. (31) in simplifying the analysis of the nonlinear Vlasov-Poisson equations (1) and (2) will become apparent in the remainder of Sec. III. In this regard, it is important to recognize that beam distribution functions typically tend to be smooth, differentiable functions with respect to $z$ and $p_{z}$, rather than have singular derivatives, as does the distribution in Eq. (31) with respect to $p_{z}$. For this reason, in Sec. IV we describe a more general formulation of nonlinear, coherent structures and traveling-wave solutions to the Vlasov-Poisson equations (1) and (2) that accommodates smooth distribution functions $F_{b}$ [see Eqs. (76) and (89) in Sec. IV]. It is certainly the case that the detailed shape of the distribution function in phase space $\left(z, p_{z}\right)$ can have an important influence on beam stability properties and collective excitations $[1,14,15]$. Nonetheless, what is also noteworthy is the fact that the waterbag model does a very good job in describing the nonlinear evolution of the beam line density, beam flow velocity, and beam particle pressure in circumstances where the heat low $Q_{b}$ can be treated as negligibly small [compare Eqs. (27) and (41), and see related discussions].

For the choice of waterbag distribution in Eq. (31), we calculate several macroscopic fluid quantities [see also Eqs. (11)-(15)] corresponding to line density

$$
\lambda_{b}=\int d p_{z} F_{b}=A m_{b}\left(V_{b}^{+}-V_{b}^{-}\right),
$$

axial flow velocity

$$
V_{b}=\lambda_{b}^{-1} \int d p_{z} v_{z} F_{b}=\frac{1}{2}\left(V_{b}^{+}+V_{b}^{-}\right),
$$

beam particle pressure

$$
\begin{aligned}
P_{b} & =m_{b} \int d p_{z}\left(v_{z}-V_{b}\right)^{2} F_{b}=\frac{1}{12} m_{b}^{2} A\left(V_{b}^{+}-V_{b}^{-}\right)^{3} \\
& =\frac{1}{12 m_{b} A^{2}} \lambda_{b}^{3}
\end{aligned}
$$

and beam particle heat flow

$$
Q_{b}=m_{b} \int d p_{z}\left(v_{z}-V_{b}\right)^{3} F_{b}=0 .
$$

Note that the heat flow is exactly $Q_{b}=0$ for the choice of waterbag distribution in Eq. (31).

Making use of the dynamical equations for $V_{b}^{-}(z, t)$ and $V_{b}^{+}(z, t)$ in Eqs. (33) and (34), where $\left\langle E_{z}\right\rangle$ is defined in Eq. (2), some straightforward algebra shows that $\lambda_{b}(z, t)$, $V_{b}(z, t)$, and $P_{b}(z, t)$ evolve according to [15]

$$
\begin{gathered}
\frac{\partial}{\partial t} \lambda_{b}+\frac{\partial}{\partial z}\left(\lambda_{b} V_{b}\right)=0 \\
\lambda_{b}\left(\frac{\partial}{\partial t} V_{b}+V_{b} \frac{\partial}{\partial z} V_{b}\right)+\frac{1}{m} \frac{\partial P_{b}}{\partial z} \\
=-\lambda_{b}\left(\frac{U_{b 0}^{2}}{\lambda_{b 0}} \frac{\partial}{\partial z} \lambda_{b}+\frac{U_{b 2}^{2} r_{w}^{2}}{\lambda_{b 0}} \frac{\partial^{3} \lambda_{b}}{\partial z^{3}}\right), \\
\left(\frac{\partial}{\partial t}+V_{b} \frac{\partial}{\partial z}\right)\left(\frac{P_{b}}{\lambda_{b}^{3}}\right)=0 .
\end{gathered}
$$

Note from Eqs. (37) and (41) that $P_{b}(z, t)$ can be expressed as

$$
P_{b}(z, t)=\frac{P_{b 0}}{\lambda_{b 0}^{3}} \lambda_{b}^{3}(z, t),
$$

where $P_{b 0}=$ const and $\lambda_{b 0}=$ const represent the characteristic (e.g., average) values of the pressure and line density, respectively, of the beam particles, and $P_{b 0} / \lambda_{b 0}^{3}=$ $1 / 12 m_{b} A^{2}=$ const, where $A$ is the constant phase-space density in Eq. (31). By virtue of the fact that the heat flow $Q_{b}(z, t)=0$ exactly for the choice of distribution function $F_{b}\left(z, p_{z}, t\right)$ in Eq. (31), it is not surprising that Eqs. (39)(42) are identical to the macroscopic fluid equations (16), (20) and (30), obtained in Sec. II B, where Eq. (30) has made the assumption of negligible heat flow in Eq. (27). 
Note here that $N_{b}(z, t)$ and $\lambda_{b}(z, t)$ are related by $N_{b}(z, t)=\lambda_{b}(z, t) / \lambda_{b 0}$.

For the present purposes, we introduce the effective thermal speed $U_{b T}$ associated with the waterbag distribution in Eq. (31) defined by

$$
U_{b T}^{2}=\frac{3 P_{b 0}}{\lambda_{b 0} m_{b}},
$$

and the normalized (dimensionless) fluid quantities $\eta(z, t)$ and $U(z, t)$ defined by

$\eta=N_{b}-1=\frac{\lambda_{b}-\lambda_{b 0}}{\lambda_{b 0}}, \quad U=\frac{V_{b}}{\left(U_{b 0}^{2}+U_{b T}^{2}\right)^{1 / 2}}$.

In Eq. (44), $\left(U_{b 0}^{2}+U_{b T}^{2}\right)^{1 / 2}$ is the effective sound speed associated with the geometric factor $g_{0}$ and the thermal speed $U_{b T}$. Furthermore, we introduce the scaled (dimensionless) time variable $T$ and spatial variable $Z$ defined by

$T=\left(\frac{U_{b 0}^{2}+U_{b T}^{2}}{U_{b 2}^{2}}\right) \frac{U_{b 2} t}{r_{w}}, \quad Z=\left(\frac{U_{b 0}^{2}+U_{b T}^{2}}{U_{b 2}^{2}}\right)^{1 / 2} \frac{z}{r_{w}}$.

Making use of the macroscopic equations (39), (40), and (42), and the definitions in Eqs. (43)-(45), it is straightforward to show that the continuity equation (39) and force balance equation (40) reduce in dimensionless variables exactly to

$$
\begin{gathered}
\frac{\partial}{\partial T} \eta+\frac{\partial}{\partial Z}(U+\eta U)=0 \\
\frac{\partial}{\partial T} U+\frac{\partial}{\partial Z}\left(\frac{1}{2} U^{2}+\eta+\frac{1}{2} \frac{U_{b T}^{2}}{U_{b 0}^{2}+U_{b T}^{2}} \eta^{2}+\frac{\partial^{2}}{\partial Z^{2}} \eta\right)=0 .
\end{gathered}
$$

The fluid description in scaled variables provided by Eqs. (46) and (47) is exactly equivalent to the kinetic description provided by Eqs. (1) and (2) for the choice of waterbag distribution in Eq. (31).

\section{B. Coherent nonlinear traveling-wave and traveling-pulse solutions}

Within the context of the present 1D model, Eqs. (46) and (47) can be used to investigate detailed properties of collective excitations over a wide range of system parameters. For example, in the weakly nonlinear regime, for small-amplitude disturbances moving near the sound speed $\left(U_{b 0}^{2}+U_{b T}^{2}\right)^{1 / 2}$, Eqs. (46) and (47) can be shown to reduce to the Korteweg-deVries equation [15], which exhibits the generation and interaction of coherent structures (solitons) for a wide range of initial density perturbations $\eta(Z, T=0) \neq 0$ [22]. While the analysis in
Ref. [15] has several interesting features, the results are limited to the weakly nonlinear regime where $|\eta| \ll 1$ and $|U| \ll 1$.

In this paper, we examine Eqs. (46) and (47) in circumstances where there are no a priori restrictions to small amplitude, i.e., $\eta=\left(\lambda_{b}-\lambda_{b 0}\right) / \lambda_{b 0}$ is allowed to be of order unity, as long as $\lambda_{b} / \lambda_{b 0}>0$, which corresponds to $\eta>-1$. Furthermore, we look for solutions to Eqs. (46) and (47) that depend on $Z$ and $T$ exclusively through the variables $Z^{\prime}=Z-M T$ and $T^{\prime}=T$, where $M=$ const is the normalized pulse speed measured in units of the sound speed $\left(U_{b 0}^{2}+U_{b T}^{2}\right)^{1 / 2}$. Making use of $\partial / \partial Z=\partial / \partial Z^{\prime}$ and $\partial / \partial T=\partial / \partial T^{\prime}-M \partial / \partial Z^{\prime}$ and looking for time-stationary solutions $\left(\partial / \partial T^{\prime}=0\right)$ in the frame of reference moving with normalized velocity $M=$ const, Eqs. (46) and (47) for $\eta\left(Z^{\prime}\right)$ and $U\left(Z^{\prime}\right)$ become

$$
\frac{\partial}{\partial Z^{\prime}}[(-M+U) \eta+U]=0,
$$

$\frac{\partial}{\partial Z^{\prime}}\left[\frac{1}{2} U^{2}-M U+\eta+\frac{1}{2} \frac{U_{b T}^{2}}{U_{b 0}^{2}+U_{b T}^{2}} \eta^{2}+\frac{\partial^{2} \eta}{\partial Z^{\prime 2}}\right]=0$.

Integrating with respect to $Z^{\prime}$, Eqs. (48) and (49) give

$$
-M \eta+(1+\eta) U=\text { const, }
$$

$\frac{1}{2} U^{2}-M U+\eta+\frac{1}{2} \frac{U_{b T}^{2}}{U_{b 0}^{2}+U_{b T}^{2}} \eta^{2}+\frac{\partial^{2} \eta}{\partial Z^{\prime 2}}=\mathrm{const}$,

which relate $\eta\left(Z^{\prime}\right)$ and $U\left(Z^{\prime}\right)$, where $Z^{\prime}=Z-M T$.

The solutions for $\eta\left(Z^{\prime}\right)$ and $U\left(Z^{\prime}\right)$ to Eqs. (50) and (51) depend on the values of the constants in Eqs. (50) and (51). For the present purposes we consider boundary conditions such that $U=0$ when $\eta=0$, and $\eta^{\prime \prime}=0$ when $U=0$ and $\eta=0$. In this case the values of the constants in Eqs. (50) and (51) are zero, which gives

$$
U=M \frac{\eta}{1+\eta} .
$$

$$
\frac{\partial^{2} \eta}{\partial Z^{\prime 2}}+\left\{\frac{1}{2}(U-M)^{2}-\frac{1}{2} M^{2}+\eta+\frac{1}{2} \frac{U_{b T}^{2}}{U_{b 0}^{2}+U_{b T}^{2}} \eta^{2}\right\}=0 .
$$

Substituting Eq. (52) into Eq. (53), we obtain

$$
\frac{\partial^{2} \eta}{\partial Z^{\prime 2}}+\left\{\frac{1}{2} M^{2}\left[\frac{1}{(1+\eta)^{2}}-1\right]+\eta+\frac{1}{2} \frac{U_{b T}^{2}}{U_{b 0}^{2}+U_{b T}^{2}} \eta^{2}\right\}=0,
$$


which is a second-order nonlinear differential equation for the perturbation in line density $\eta\left(Z^{\prime}\right)=\left[\lambda_{b}\left(Z^{\prime}\right)-\lambda_{b 0}\right] / \lambda_{b 0}$, where $Z^{\prime}=Z-M T$. Some straightforward algebraic manipulation shows that Eq. (54) can be expressed in the equivalent form

$$
\frac{\partial^{2} \eta}{\partial Z^{\prime 2}}=-\frac{\partial}{\partial \eta} V(\eta)
$$

where $V(\eta)$ is the effective potential defined by

$$
V(\eta)=\frac{1}{2} \frac{\eta^{2}}{1+\eta}\left\{\epsilon_{T} \eta^{2}+\left(1+\epsilon_{T}\right) \eta+\left(1-M^{2}\right)\right\},
$$

and the dimensionless parameter $\epsilon_{T}$, defined by

$$
\epsilon_{T}=\frac{1}{3}\left(\frac{U_{b T}^{2}}{U_{b 0}^{2}+U_{b T}^{2}}\right),
$$

is a measure of the longitudinal thermal speed of the beam particles.

Equations (55) and (56) can be used to determine the solutions for $\eta\left(Z^{\prime}\right)$ for a broad range of dimensionless parameters $\epsilon_{T}$ and $M$. Furthermore, Eqs. (55) and (56) have been obtained from Eqs. (50) and (51) for the special class of boundary conditions where $U=0$ and $\eta^{\prime \prime}=0$ when $\eta=$ 0 [see discussion prior to Eqs. (52) and (53)]. Indeed, we will show below that Eqs. (55) and (56) support two classes of solutions consistent with these boundary conditions. These correspond to: (i) localized (pulselike) soliton solutions when $M^{2}>1$, satisfying $\eta\left(Z^{\prime}= \pm \infty\right)=0$, $U\left(Z^{\prime}= \pm \infty\right)=0$, and $\left[\partial^{2} \eta / \partial Z^{\prime 2}\right]_{Z^{\prime}= \pm \infty}=0$; and (ii) nonlinear periodic traveling-wave solutions when $M^{2}<1$, with $\eta\left(Z^{\prime}\right)=\eta\left(Z^{\prime}+L\right)$, and $\eta\left(Z^{\prime}=0\right)=0, U\left(Z^{\prime}=0\right)=0$, and $\left[\partial^{2} \eta / \partial Z^{\prime 2}\right]_{Z^{\prime}=0}=0$.

In general, the effective potential $V(\eta)$ in Eq. (57) can be expressed as

$$
V(\eta)=\frac{1}{2} \frac{\eta^{2}}{1+\eta} \epsilon_{T}\left[\left(\eta-\eta^{+}\right)\left(\eta-\eta^{-}\right)\right]
$$

where

$\eta^{ \pm}=\frac{1}{2}\left\{-\left(1+\frac{1}{\epsilon_{T}}\right) \pm\left[\left(1+\frac{1}{\epsilon_{T}}\right)^{2}+\frac{4}{\epsilon_{T}}\left(M^{2}-1\right)^{1 / 2}\right]\right\}$.

In Eqs. (58) and (59), $\epsilon_{T}$ is restricted to the range $0<\epsilon_{T}<1 / 3$, and $M^{2}$ can satisfy $M^{2}>1$ or $M^{2}<1$. Examination of Eq. (59) shows that

$$
\eta^{-}<-1, \quad \eta^{+}>-1,
$$

for all allowed values of $\epsilon_{T}$ and $M^{2}$. Furthermore, it is also clear from Eq. (59) that

$$
\left\{\begin{array}{l}
\eta^{+}>0, \quad \text { for } M^{2}>1 \\
\eta^{+}<0, \quad \text { for } M^{2}<1
\end{array}\right.
$$

Recall that $\eta=\left(\lambda_{b}-\lambda_{b 0}\right) / \lambda_{b 0}$. Then $\lambda_{b} / \lambda_{b 0} \geq 0$ implies that $\eta \geq-1$ is the region of interest physically for solutions to Eq. (55).

Note that Eq. (55) has the form of a dynamical equation of motion, with $\eta$ playing the role of displacement, $Z^{\prime}$ playing the role of time, and $V(\eta)$ playing the role of an effective potential, or so-called pseudopotential. Multiplying Eq. (55) by $\partial \eta / \partial Z^{\prime}$ and integrating, we obtain

$$
\frac{1}{2}\left(\frac{\partial \eta}{\partial Z^{\prime}}\right)^{2}+V(\eta)=E=\mathrm{const}
$$

Equation (62) plays the role of an effective energy conservation constraint, and can be integrated to determine $\eta\left(Z^{\prime}\right)$ for the pseudopotential $V(\eta)$ defined in Eq. (58). We now examine solutions to Eq. (62) for the two cases identified earlier: $M^{2}<1$ and $-1<\eta^{+}<0$; and $M^{2}>1$ and $\eta^{+}>0$.

\section{Nonlinear traveling-wave solutions $\left(M^{2}<\mathbf{1}\right.$ and $\left.-\mathbf{1}<\eta^{+}<\mathbf{0}\right)$}

Figure 1 shows a schematic plot of $V(\eta)$ versus $\eta$ for the case where $M^{2}<1$ and $-1<\eta^{+}<0$. For the purposes of illustration, the values of the specific parameters in Fig. 1 have been chosen to be $M^{2}=0.09$ and $\epsilon_{T}=4 / 15$ in plotting $V(\eta)$ versus $\eta$. The corresponding values of $\eta^{+}$, $\eta_{m}$ and $V\left(\eta_{m}\right)$ are $\eta^{+}=-0.882, \quad \eta_{m}=-0.715$, and $V\left(\eta_{m}\right)=0.126$. For different choices of values for $\epsilon_{T}$ and $M^{2}<1$, the shape of the $V(\eta)$ versus $\eta$ curve is qualitatively similar to that shown in Fig. 1. Referring to Fig. 1, when the effective energy $E$ (the red horizontal line in Fig. 1 lies in the interval $0<E<V\left(\eta_{m}\right)$, Eq. (66) supports nonlinear periodic solutions for $\eta\left(Z^{\prime}\right)$ that oscillate as a function of $Z^{\prime}$. Here $V\left(\eta_{m}\right)$ is the local maximum of $V(\eta)$, which occurs at $\eta=\eta_{m}$ in Fig. 1. Depending on system parameters, these nonlinear traveling-wave

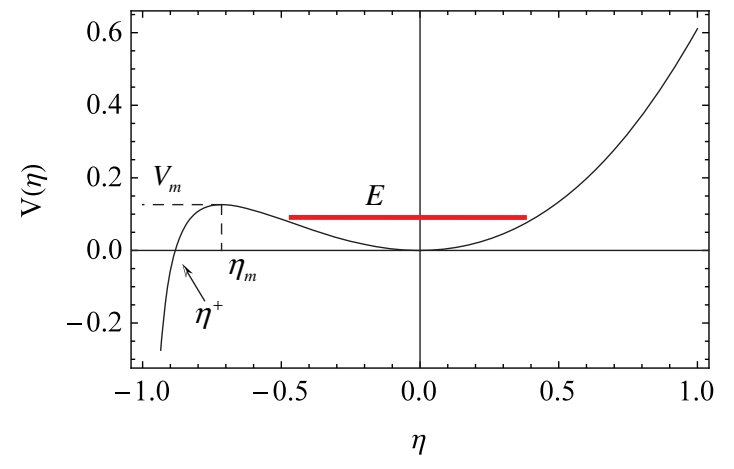

FIG. 1. Illustrative plot of $V(\eta)$ versus $\eta$ obtained from Eq. (56) for $M^{2}=0.09$ and $\epsilon_{T}=4 / 15$. Here, $\eta^{+}=-0.882, \eta_{m}=-0.715$ and $V\left(\eta_{m}\right)=0.126$. 

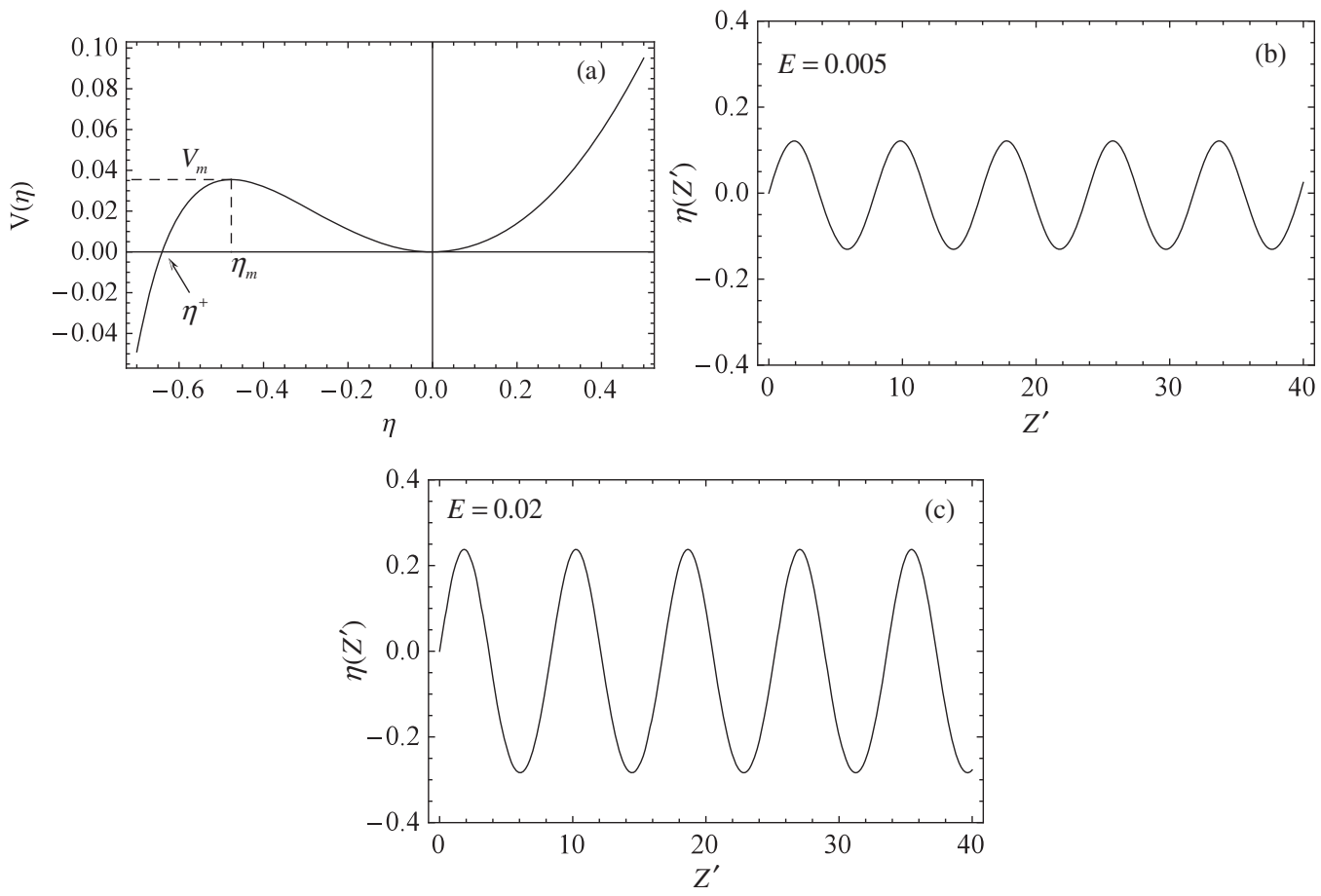

FIG. 2. For $M^{2}=0.36, \epsilon_{T}=0, \eta^{+}=-0.64, \eta_{m}=-0.476$ and $V\left(\eta_{m}\right)=0.0355$, plots are shown for (a) $V(\eta)$ versus $\eta$; (b) $\eta\left(Z^{\prime}\right)$ versus $Z^{\prime}$ for $\eta^{\prime}(0)=0.1$ and $E=(1 / 2)\left[\eta^{\prime}(0)\right]^{2}=0.005$; and (c) $\eta\left(Z^{\prime}\right)$ versus $Z^{\prime}$ for $\eta^{\prime}(0)=0.2$ and $E=(1 / 2)\left[\eta^{\prime}(0)\right]^{2}=0.02$.
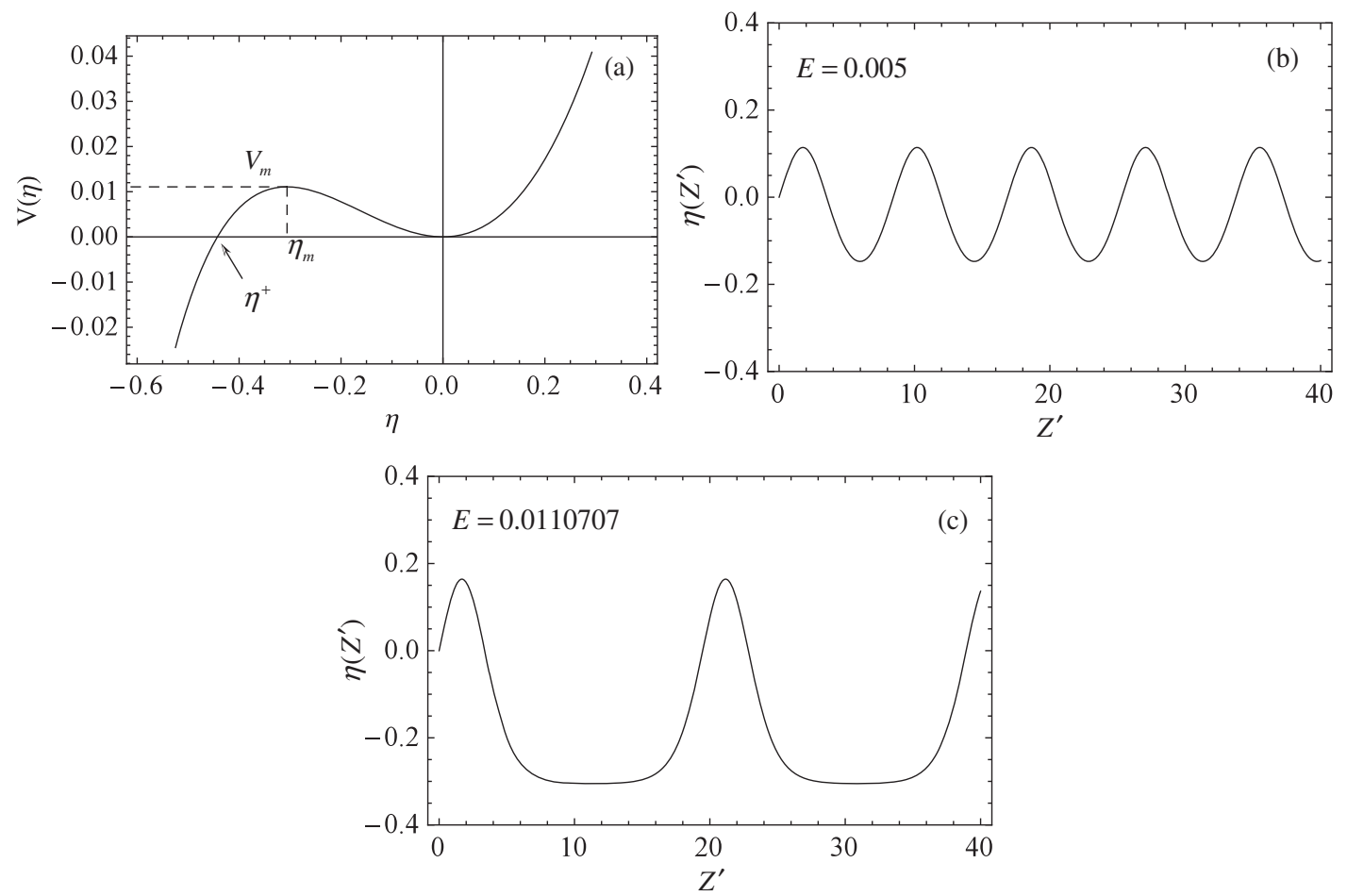

FIG. 3. For $M^{2}=0.36, \epsilon_{T}=0.8, \eta^{+}=-0.442, \eta_{m}=-0.306$ and $V\left(\eta_{m}\right)=0.0111$, plots are shown for (a) $V(\eta)$ versus $\eta$; (b) $\eta\left(Z^{\prime}\right)$ versus $\quad Z^{\prime} \quad$ for $\quad \eta^{\prime}(0)=0.1 \quad$ and $\quad E=(1 / 2)\left[\eta^{\prime}(0)\right]^{2}=0.005 ; \quad$ and $\quad$ (c) $\quad \eta\left(Z^{\prime}\right) \quad$ versus $\quad Z^{\prime} \quad$ for $\quad \eta^{\prime}(0)=0.1488$ and $E=(1 / 2)\left[\eta^{\prime}(0)\right]^{2}=0.0110707$. 

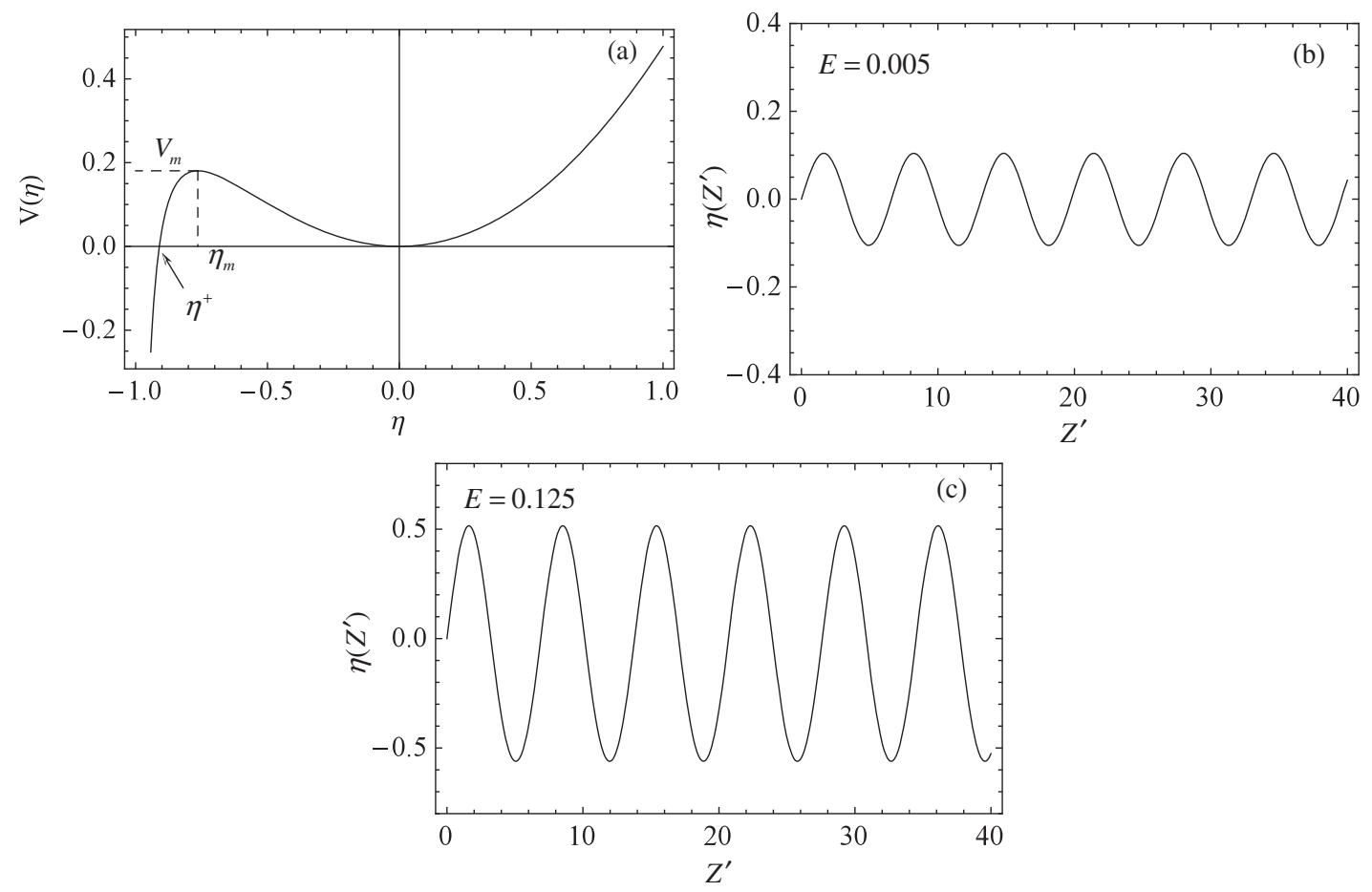

FIG. 4. For $M^{2}=0.09, \epsilon_{T}=0, \eta^{+}=-0.91, \eta_{m}=-0.764$ and $V\left(\eta_{m}\right)=0.181$, plots are shown for (a) $V(\eta)$ versus $\eta$; (b) $\eta\left(Z^{\prime}\right)$ versus $Z^{\prime}$ for $\eta^{\prime}(0)=0.1$ and $E=(1 / 2)\left[\eta^{\prime}(0)\right]^{2}=0.005$; and (c) $\eta\left(Z^{\prime}\right)$ versus $Z^{\prime}$ for $\eta^{\prime}(0)=0.5$ and $E=(1 / 2)\left[\eta^{\prime}(0)\right]^{2}=0.125$.
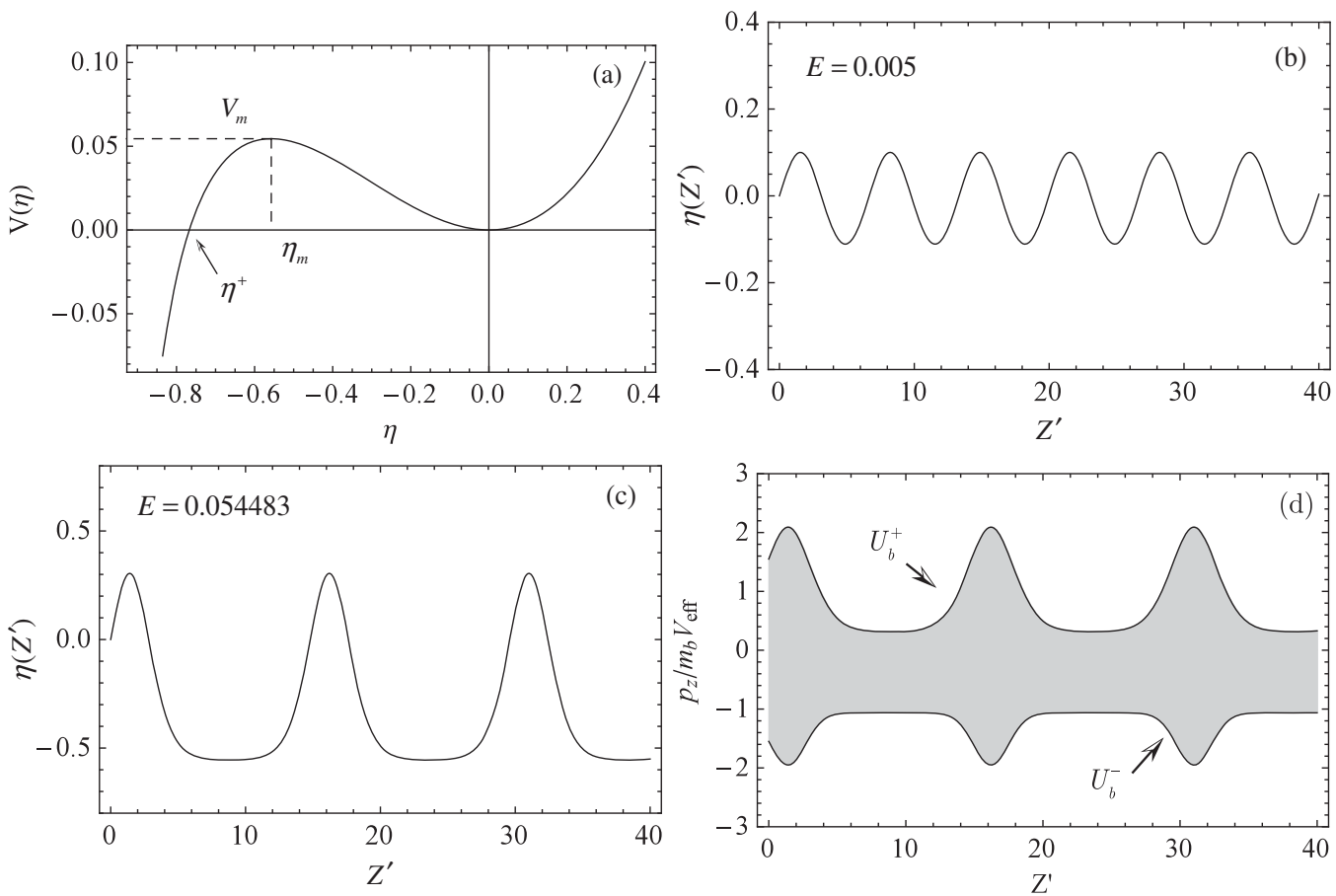

FIG. 5. For $M^{2}=0.09, \epsilon_{T}=0.8, \eta^{+}=-0.767, \eta_{m}=-0.557$ and $V\left(\eta_{m}\right)=0.0545$, plots are shown for (a) $V(\eta)$ versus $\eta$; (b) $\eta\left(Z^{\prime}\right)$ versus $\quad Z^{\prime} \quad$ for $\quad \eta^{\prime}(0)=0.1 \quad$ and $\quad E=(1 / 2)\left[\eta^{\prime}(0)\right]^{2}=0.005 ; \quad$ and $\quad\left(\right.$ c) $\quad \eta\left(Z^{\prime}\right) \quad$ versus $\quad Z^{\prime} \quad$ for $\quad \eta^{\prime}(0)=0.3301 \quad$ and $E=(1 / 2)\left[\eta^{\prime}(0)\right]^{2}=0.054483$. Shown in (d) is a self-consistent phase-space plot for the waterbag distribution [Eq. (31)] for the choice of system parameters in Fig. 5(c). 
solutions can have large amplitude, representing a significant modulation in beam line density.

Referring to the discussion preceding Eq. (58), the boundary conditions used to derive Eqs. (55) and (56) from Eqs. (50) and (51) correspond to

$$
\eta(0)=0=\eta^{\prime \prime}(0)
$$

for the class of nonlinear periodic wave solutions obtained from Eq. (62) when $M^{2}<1$ and $-1<\eta^{+}<0$. Furthermore, from Fig. 1 and Eq. (62), we note that $V(\eta=0)=0$ and the effective energy $E$ can be expressed as

$$
E=\frac{1}{2}\left[\eta^{\prime}(0)\right]^{2} .
$$

Typical numerical solutions for $\eta\left(Z^{\prime}\right)$, obtained by integrating Eq. (62) with $V(\eta)$ specified by Eq. (58), are illustrated in Figs. 2-5 for several values of $M^{2}<1$ and $\epsilon_{T}$, and different values of effective energy level $E$. These correspond to: $M^{2}=0.36, \epsilon_{T}=0, E=0.005$, and $E=$ 0.0110707 (Fig. 2); $M^{2}=0.36, \epsilon_{T}=4 / 15, E=0.005$, and $E=0.020$ (Fig. 3); $M^{2}=0.09, \epsilon_{T}=0, E=0.005$, and $E=0.054483$ (Fig. 4); $M^{2}=0.09, \epsilon_{T}=4 / 15$, $E=0.005$, and $E=0.125$ (Fig. 5). Close examination of Figs. 2-5 shows several interesting trends. First, for smaller values of $M^{2}$, the potential wells are deeper and broader (compare Figs. 2a and 4a, and Figs. 3a and 5a); and for smaller values of $\epsilon_{T}$, the potential wells are deeper (compare Figs. 2a and 3a, and Figs. 4a and 5a). Furthermore, the nonlinear wave amplitude tends to be larger for smaller values of $M^{2}$ (compare Figs. 2a and 3a, and Figs. 4a and 5a), whereas the wavelength dependence on $M^{2}$ and $\epsilon_{T}$ tends to be relatively weak (compare Figs. 2, $3,4,5)$. In any case, for $M^{2}<1$, it is clear from Figs. 1-5 that Eqs. (62) and (58) support a broad class of nonlinear traveling-wave solutions for the theoretical model developed here, based on the 1D kinetic waterbag model for intense beam propagation. Indeed, the modulation of the beam line density is about $\pm 50 \%$ for the system parameters in Figs. 4(c) and 5(c).

\section{Nonlinear traveling-pulse (soliton) solutions $\left(M^{2}>1\right.$ and $\left.\eta^{+}>0\right)$}

We now consider Eqs. (62) and Eq. (58) [or equivalently, Eq. (56)] in circumstances where $M^{2}>1$ and $\eta^{+}>0$. In this case, the effective potential has the qualitative shape illustrated in Fig. 6, which has been plotted for the choice of parameters $M^{2}=9$ and $\epsilon_{T}=1 / 30$. The physically allowed, localized pulse solutions (soliton solutions) corresponds to the energy level

$$
E=0,
$$

which is the red horizontal line in Fig. 6, and boundary conditions

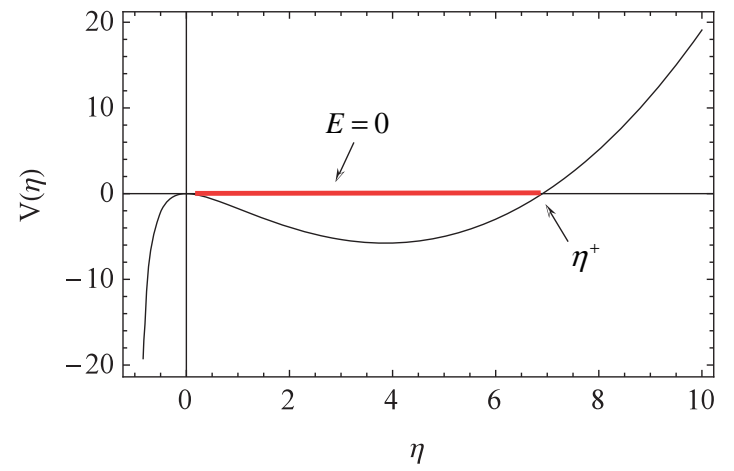

FIG. 6. Illustrative plot of $V(\eta)$ versus $\eta$ obtained from Eq. (56) for $M^{2}=9$ and $\epsilon_{T}=1 / 50$. Here, $\eta^{+}=6.908$, and the energy level $E=0$ corresponds to soliton solutions with maximum amplitude $\eta^{+}=6.908$.

$$
\eta\left(Z^{\prime}= \pm \infty\right)=0=\eta^{\prime \prime}\left(Z^{\prime}= \pm \infty\right)
$$

discussed prior to Eq. (58). Referring to Fig. 6, when Eq. (62) is integrated forward from $Z^{\prime}=-\infty$ where $\eta=0$, the perturbed line density, $\eta$ increases monotonically through positive values to a maximum amplitude $\eta^{+}$(the soliton amplitude) and then decreases monotonically to $\eta=0$ when $Z^{\prime}=+\infty$. The regime where $M^{2}>1$ by a sufficiently large amount corresponds to a strongly nonlinear regime where the density compression is large with $\eta^{+}>1$. On the other hand, when $M^{2}-1=\epsilon$ is small
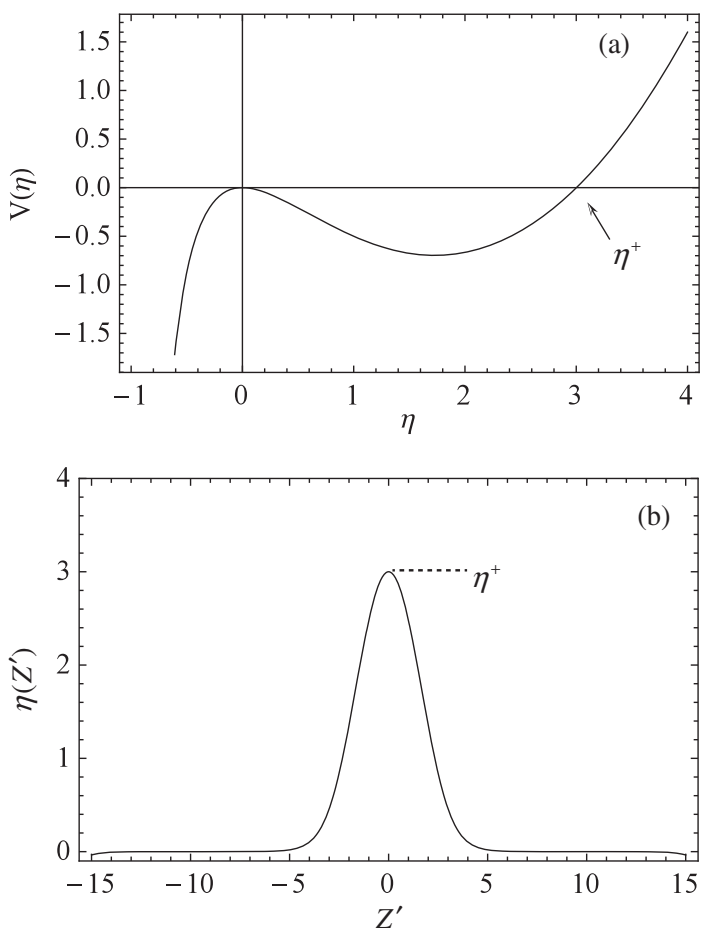

FIG. 7. Plots of (a) $V(\eta)$ versus $\eta$; and (b) $\eta\left(Z^{\prime}\right)$ versus $Z^{\prime}$, obtained from Eqs. (56) and (62) for $M^{2}=4, \epsilon_{T}=0$ and $E=0$, corresponding to soliton amplitudes $\eta^{+}=3.0$. 

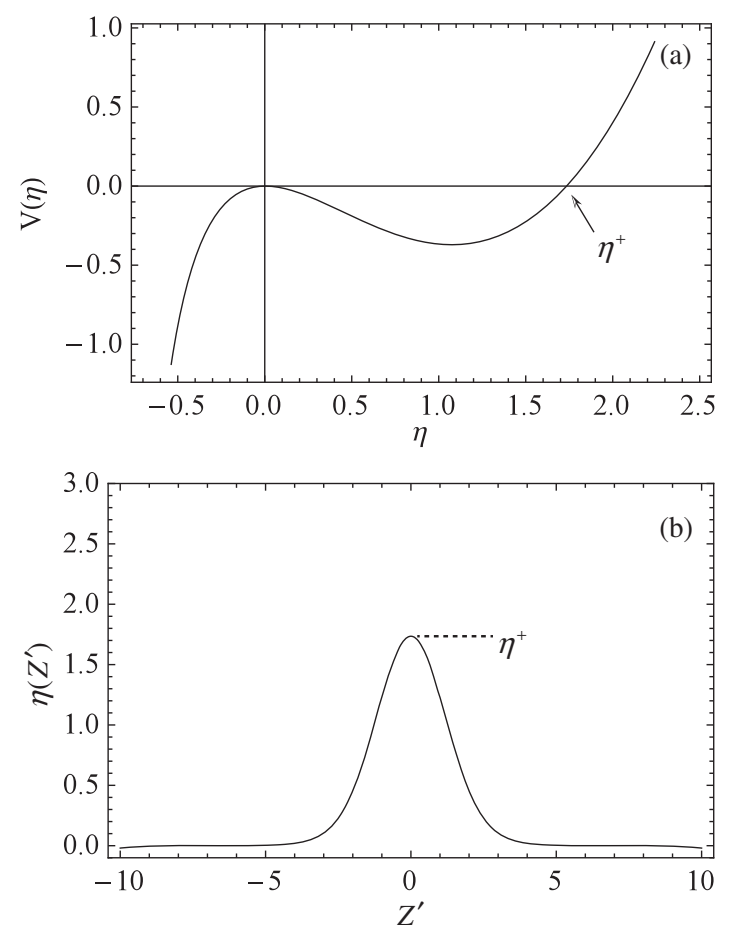

FIG. 8. Plots of (a) $V(\eta)$ versus $\eta$; and (b) $\eta\left(Z^{\prime}\right)$ versus $Z^{\prime}$, obtained from Eqs. (56) and (62) for $M^{2}=4, \epsilon_{T}=4 / 15$ and $E=0$, corresponding to soliton amplitudes $\eta^{+}=1.735$.

with $0<\epsilon \ll 1$, the soliton amplitude is correspondingly small. This will become apparent from the numerical solutions to Eqs. (62) and (58) consistent with Eqs. (65) and (66) presented later in this section in Figs. 7-10.

Typical numerical solutions to Eqs. (62) and (58), subject to Eqs. (65) and (66), are illustrated in Figs. 7-10 for several values of $M^{2}>1$ and $\epsilon_{T}$. These correspond to: $M^{2}=4$ and $\epsilon_{T}=0$ (Fig. 7); $M^{2}=4$ and $\epsilon_{T}=4 / 15$ (Fig. 8); $M^{2}=1.2$ and $\epsilon_{T}=0$ (Fig. 9); and $M^{2}=1.2$ and $\epsilon_{T}=4 / 15$ (Fig. 10). Close examination of Figs. 7-10 shows that the soliton amplitude increases with increasing $M^{2}$ (compare Figs. 7 and 8 with Figs. 9 and 10), reaching a highly nonlinear regime with $\eta^{+}=3.0$ in Fig. 7 and $\eta^{+}=$ 1.735 in Fig. 8, where $M^{2}=4$. In contrast, the soliton width trends show a relatively weak dependence on longitudinal velocity spread, as measured by $\epsilon_{T}$ (compare Fig. 8 with Fig. 7, and Fig. 10 with Fig. 9). It is clear from Figs. 7-10 that the soliton solutions to Eqs. (62) and (58) exhibit a strong nonlinear dependence on $M^{2}$, and can correspond to highly compressed line density for sufficiently large $M^{2}$.

In the special circumstances where $M^{2}$ exceeds 1 by a small amount, i.e., $M^{2}=1+\Delta$ where $0<\Delta \ll 1$. it is readily shown that Eq. (54) can be approximated for small $\eta$ by

$$
\frac{\partial^{2} \eta}{\partial Z^{\prime 2}}+\left\{\left[\frac{3}{2} M^{2}+\frac{3}{2} \epsilon_{T}\right] \eta-\left(M^{2}-1\right)\right\} \eta=0 .
$$
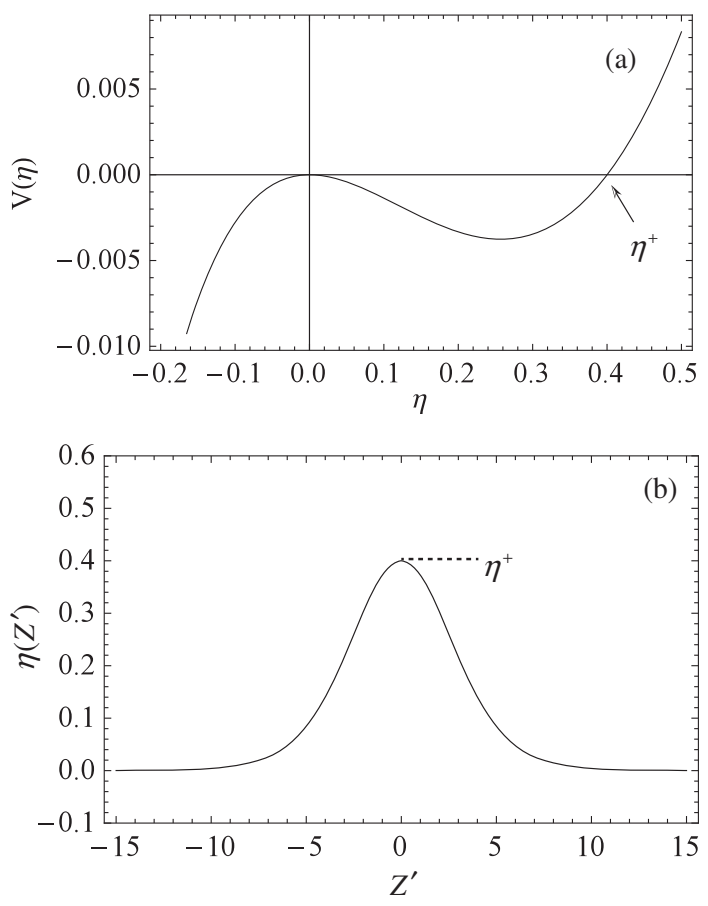

FIG. 9. Plots of (a) $V(\eta)$ versus $\eta$; and (b) $\eta\left(Z^{\prime}\right)$ versus $Z^{\prime}$, obtained from Eqs. (56) and (62) for $M^{2}=1.2, \epsilon_{T}=0$ and $E=0$, corresponding to soliton amplitudes $\eta^{+}=0.4$.

Equation (67) can be solved exactly for $\eta\left(Z^{\prime}\right)=$ $\lambda_{b}\left(Z^{\prime}\right) / \lambda_{b 0}-1$ to give

$\eta\left(Z^{\prime}\right)=\left(\frac{M^{2}-1}{M^{2}+\epsilon_{T}}\right) \operatorname{sech}^{2}\left[\frac{1}{2}\left(M^{2}-1\right)^{1 / 2}(Z-M T)\right]$.

Note that the soliton amplitude in Eq. (68) is small for $M^{2}=1+\Delta$ with $\Delta \ll 1$. Also, the $\operatorname{sech}^{2}\{\cdots\}$ pulse shape in Eq. (68) is similar to the soliton pulse shape obtained from the Korleweg-deVries equation in the weakly nonlinear regime [15].

Finally, it should be noted that the oscillatory solutions obtained from Eqs. (58) and (62) when $M^{2}>1$ and the energy level $E$ in Fig. 6 is negative with $V_{\min }<E<0$ are not considered here. These solutions are unphysical because they oscillate about a positive nonzero average value of $\bar{\eta}=\bar{\lambda}_{b} / \lambda_{b 0}-1>0$, rather than oscillate about $\bar{\eta}=\bar{\lambda}_{b} / \lambda_{b 0}-1 \approx 0$, as occurs in Figs. $2-5$ when $M^{2}<1$.

In concluding Sec. III, it should be noted that once $\eta\left(Z^{\prime}\right)$ is determined from Eq. (62) for specified system parameters, then Eqs. (35), (36), and (52) can readily be used to develop plots of the nonlinearly distorted phase-space boundaries $V_{b}^{+}$and $V_{b}^{-}$, both for the case of nonlinear traveling wave solutions (Figs. 1-5) and for nonlinear traveling soliton solutions (Figs. 6-10). Normalizing $V_{b}^{+}$, $V_{b}^{-}$, and $V_{b}$ to the effective sound speed $V_{\text {eff }}=\left(U_{b 0}^{2}+\right.$ $\left.U_{b T}^{2}\right)^{1 / 2}$ according to $U_{b}^{+}=V_{b}^{+} / V_{\text {eff }}, U_{b}^{-}=V_{b}^{-} / V_{\text {eff }}$, and $U=V_{b} / V_{\text {eff }}$, Eqs. (35), (36), and (52) give $\eta+1=$ 

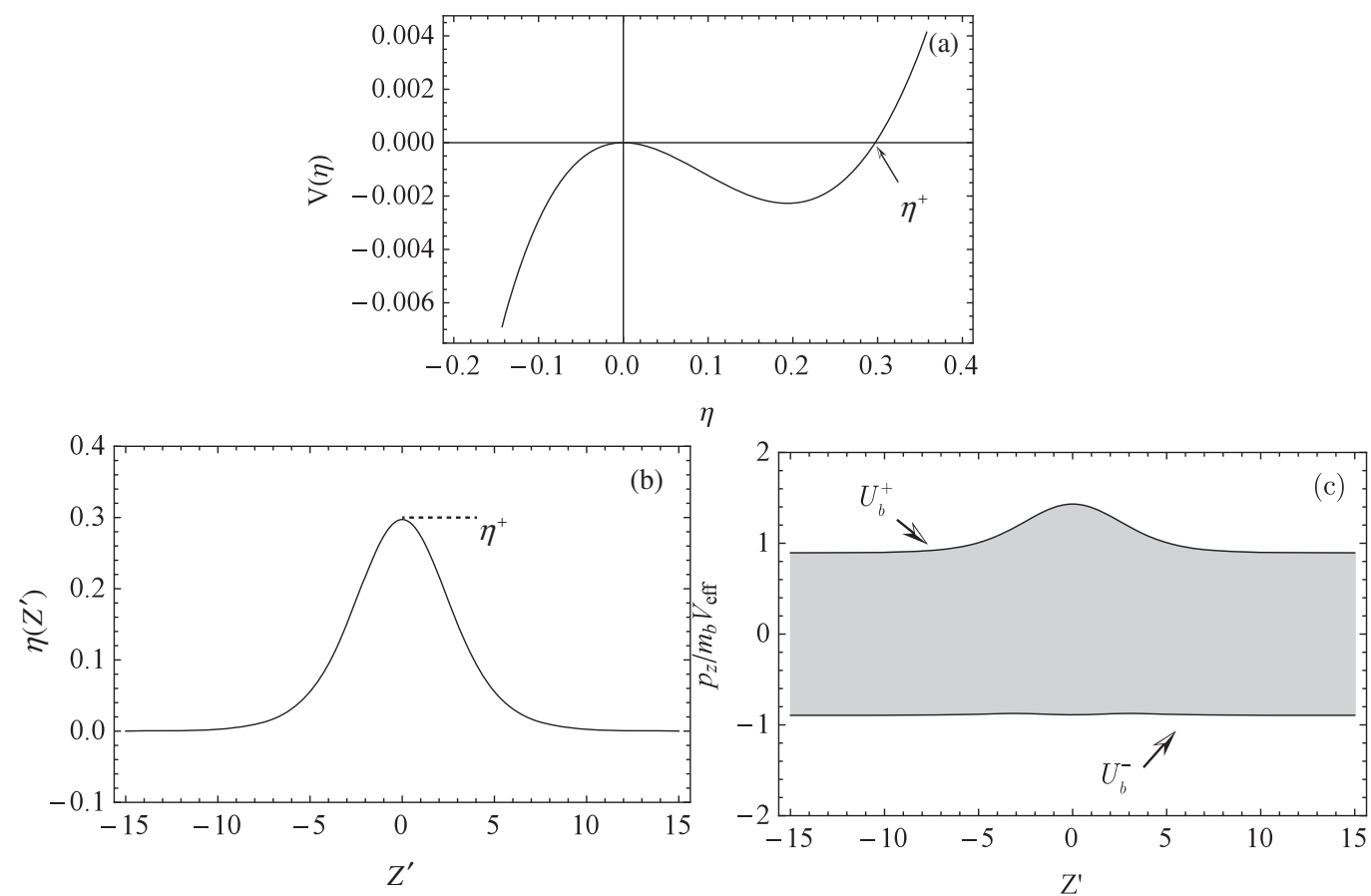

FIG. 10. Plots of (a) $V(\eta)$ versus $\eta$; and (b) $\eta\left(Z^{\prime}\right)$ versus $Z^{\prime}$, obtained from Eqs. (56) and (62) for $M^{2}=1.2, \epsilon_{T}=4 / 15$ and $E=0$, corresponding to soliton amplitudes $\eta^{+}=0.297$. Shown in (c) is a self-consistent phase-space plot for the waterbag distribution [Eq. (31)] for the choice of system parameters in Fig. 10(b).

$\left(A m_{b} V_{\text {eff }} / \lambda_{b 0}\right)\left(U_{b}^{+}-U_{b}^{-}\right), \quad$ and $\quad U=\left(U_{b}^{+}+U_{b}^{-}\right) / 2=$ $M \eta /(1+\eta)$. For example, for the choice of system parameters and nonlinear traveling-wave solution for $\eta\left(Z^{\prime}\right)$ illustrated in Fig. 5(c), shown in Fig. 5(d) is the corresponding self-consistent phase-space plot for the waterbag distribution, which shows clearly how the boundary curves $U_{b}^{+}\left(Z^{\prime}\right)$ and $U_{b}^{-}\left(Z^{\prime}\right)$ evolve nonlinearly as the beam line density alternately compresses and decompresses as shown in Fig. 5(c). By contrast, for the choice of system parameters and compressive soliton solution for $\eta\left(Z^{\prime}\right)$ illustrated in Fig. 10(b), shown in Fig. 10(c) is the corresponding phase-space plot for the waterbag distribution, which shows how the boundary curves $U_{b}^{+}\left(Z^{\prime}\right)$ and $U_{b}^{-}\left(Z^{\prime}\right)$ evolve as the beam line density experiences a single soliton compression with moderately large amplitude.

\section{COHERENT NONLINEAR STRUCTURES OBTAINED FROM FULLY KINETIC G-FACTOR MODEL}

The kinetic waterbag model developed in Sec. III of this paper has clearly demonstrated the rich variety of coherent nonlinear structures supported by the 1D kinetic model based on Eqs. (9) and (10) [or equivalently, Eqs. (1) and (2)] for the specific choice of waterbag distribution $F_{b}\left(z, p_{z}, t\right)$ in Eq. (31). In this section, we examine solutions to Eqs. (9) and (10) for an even broader class of distribution functions $F_{b}\left(z, p_{z}, t\right)$, recognizing that Eqs. (9) and (10) are Galilean invariant. That is, if we transform variables to a frame of reference moving with constant longitudinal velocity $V_{0}=$ const according to $z^{\prime}=z-V_{0} t, p_{z}^{\prime}=p_{z}-m_{b} V_{0}, t^{\prime}=t$, then in the new dynamical variables $\left(z^{\prime}, p_{z}^{\prime}, t^{\prime}\right)$, the equations for $F_{b}\left(z^{\prime}, p_{z}^{\prime}, t^{\prime}\right)$ and $\langle\phi\rangle\left(z^{\prime}, t^{\prime}\right)$ are identical in form to Eqs. (9) and (10). Time-stationary solutions $\left(\partial / \partial t^{\prime}=0\right)$ in the new variables $\left(z^{\prime}, p_{z}^{\prime}, t^{\prime}\right)$ then correspond to undistorted traveling-wave or traveling-pulse solutions moving with constant velocity $V_{0}=$ const in the original variables $\left(z, p_{z}, t\right)$. The present analysis of Eqs. (9) and (10) parallels the original Bernstein-Greene-Kruskal (BGK) formulation of BGK solutions to the 1D Vlasov-Poisson equations $[25,26]$, except for the fact that Eq. (10), which connects $\langle\phi\rangle(z, t)$ to the line density $\lambda_{b}(z, t)$, has a very different structure than the 1D Poisson equation.

Referring to Eqs. (9) and (10), we introduce the scaled dimensionless variables $\left(Z, P_{Z}, T\right)$ defined by

$$
\begin{aligned}
Z & =\left(\frac{U_{b 0}^{2}+U_{b T}^{2}}{U_{b 2}^{2}}\right)^{\frac{1}{2}} \frac{z}{r_{w}}, \quad T=\left(\frac{U_{b 0}^{2}+U_{b T}^{2}}{U_{b 2}^{2}}\right) \frac{U_{b 2} t}{r_{w}}, \\
P_{z} & =\frac{p_{z}}{m_{b}\left(U_{b 0}^{2}+U_{b T}^{2}\right)^{1 / 2}}=\frac{v_{z}}{\left(U_{b 0}^{2}+U_{b T}^{2}\right)^{1 / 2}} \equiv V_{z}
\end{aligned}
$$

where $U_{b 0}^{2}$ and $U_{b 2}^{2}$ are defined in Eq. (3), and $U_{b T}^{2}=$ const is the longitudinal velocity spread characteristic of the distribution function $F_{b}$. We further introduce the dimensionless distribution function $\hat{F}_{b}\left(Z, P_{z}, T\right)$ defined by 


$$
\hat{F}_{b}=\lambda_{b 0}^{-1} \frac{F_{b}}{m_{b}\left(U_{b 0}^{2}+U_{b T}^{2}\right)^{1 / 2}},
$$

where $\lambda_{b 0}=$ const is the characteristic line density of the beam particles, e.g., the average value. From Eqs. (69), (70) and the definition of line density $\lambda_{b}=\int d p_{z} F_{b}$, it follows that the perturbation in line density $\eta=\lambda_{b} / \lambda_{b 0}-1$ can be expressed as

$$
\eta(Z, T)=\int d P_{z} \hat{F}_{b}\left(Z, P_{z}, T\right)-1,
$$

where the $P_{z}$ integration covers the range $-\infty<P_{z}<\infty$ in Eq. (71). Transforming variables according to Eqs. (69) and (70), and making use of Eq. (71), it is readily shown that Eqs. (9) and (10) can be expressed in the new variables as

$$
\frac{\partial \hat{F}_{b}}{\partial T}+V_{z} \frac{\partial \hat{F}_{b}}{\partial Z}-\frac{\partial \psi}{\partial Z} \frac{\partial \hat{F}_{b}}{\partial P_{z}}=0
$$

and

$$
\psi=\eta+\frac{\partial^{2} \eta}{\partial Z^{2}}
$$

where $\psi(Z, T)$ is the normalized (dimensionless) potential defined by

$$
\psi=\frac{e\langle\phi\rangle}{m_{b}\left(U_{b 0}^{2}+U_{b T}^{2}\right)^{1 / 2}} .
$$

Equations (72) and (73), where $\eta$ and $\int d P_{z} \hat{F}_{b}$ are related by Eq. (71), constitute coupled nonlinear equations describing the self-consistent evolution of the distribution function $\hat{F}_{b}\left(Z, P_{z}, T\right)$, normalized potential $\psi(Z, T)$, and normalized perturbed line density $\eta(Z, T)$. Equations (71)-(73) are fully equivalent to the original dynamical equations (9)(11), and can be used to investigate 1D kinetic properties of the nonlinear beam dynamics over a wide range of system parameters.

Keeping in mind that Eqs. (72) and (73) are Galilean invariant, if we transform Eqs. (72) and (73) from the variables $\left(Z, P_{z}, T\right)$ to a frame moving with normalized velocity $\quad M=$ const. according to $Z^{\prime}=Z-M T$, $V_{z}^{\prime}=V_{z}-M, T^{\prime}=T$, then Eqs. (72) and (73) have exactly the same form in the new variables, with $\left(Z, P_{z}, T\right)$ replaced by $\left(Z^{\prime}, P_{z}^{\prime}, T^{\prime}\right)$, i.e.,

$$
\frac{\partial \hat{F}_{b}}{\partial T^{\prime}}+V_{z}^{\prime} \frac{\partial \hat{F}_{b}}{\partial Z^{\prime}}-\frac{\partial \psi}{\partial Z^{\prime}} \frac{\partial \hat{F}_{b}}{\partial P_{z}^{\prime}}=0
$$

and

$$
\psi=\eta+\frac{\partial^{2} \eta}{\partial Z^{\prime 2}}
$$

Here, $P_{z}^{\prime}=V_{z}^{\prime}$, and $\hat{F}_{b}\left(Z^{\prime}, P_{z}^{\prime}, T^{\prime}\right)$ and $\eta\left(Z^{\prime}, T^{\prime}\right)$ are related by

$$
\eta\left(Z^{\prime}, T^{\prime}\right)=\int d P_{z}^{\prime} \hat{F}_{b}\left(Z^{\prime}, P_{z}^{\prime}, T^{\prime}\right)-1
$$

Therefore, the traveling-pulse or traveling-wave solutions that have a time-stationary profile shape in the primed variables $\left(Z^{\prime}, P_{z}^{\prime}, T^{\prime}\right)$ are determined by setting $\partial / \partial T^{\prime}=0$ in Eqs. (75)-(77).

Setting $\partial \hat{F}_{b} / \partial T^{\prime}=0$ in Eq. (75) gives for $\hat{F}_{b}\left(Z^{\prime}, P_{z}^{\prime}\right)$

$$
V_{z}^{\prime} \frac{\partial \hat{F}_{b}}{\partial Z^{\prime}}-\frac{\partial \psi}{\partial Z^{\prime}} \frac{\partial \hat{F}_{b}}{\partial P_{z}^{\prime}}=0
$$

where $\psi\left(Z^{\prime}\right)$ and $\eta\left(Z^{\prime}\right)$ solve Eq. (76), and $\eta\left(Z^{\prime}\right)$ is related to $\hat{F}_{b}\left(Z^{\prime}, P_{z}^{\prime}\right)$ by Eq. (77). We introduce the energy variable $W^{\prime}$ defined by

$$
W^{\prime}=\frac{1}{2} V_{z}^{\prime 2}+\psi\left(Z^{\prime}\right)
$$

Then the solution to Eq. (78) for $\hat{F}_{b}\left(Z^{\prime}, P_{z}^{\prime}\right)$ can be expressed exactly as

$$
\hat{F}_{b}\left(Z^{\prime}, V_{z}^{\prime}\right)=\hat{F}_{b}^{>}\left(W^{\prime}\right) \Theta\left(V_{z}^{\prime}\right)+\hat{F}_{b}^{<}\left(W^{\prime}\right) \Theta\left(-V_{z}^{\prime}\right)
$$

where $P_{z}^{\prime}=V_{z}^{\prime}$, and

$$
\Theta\left(V_{z}^{\prime}\right)= \begin{cases}1, & \text { for } V_{z}^{\prime}>0 \\ 0, & \text { for } V_{z}^{\prime}<0\end{cases}
$$

Note from Eq. (79) that

$$
d V_{z}^{\prime}= \pm d W^{\prime} /\left[2\left(W^{\prime}-\psi\right)\right]^{1 / 2},
$$

where + corresponds to $V_{z}^{\prime}>0$, and - corresponds to $V_{z}^{\prime}<0$. Substituting Eqs. (80) and (82) into Eq. (77) gives

$$
\eta=\int_{\psi}^{\infty} d W^{\prime} \frac{\left[\hat{F}_{b}^{>}\left(W^{\prime}\right)+\hat{F}_{b}^{<}\left(W^{\prime}\right)\right]}{\left[2\left(W^{\prime}-\psi\right)\right]^{1 / 2}}-1
$$

which relate the perturbation in beam line density $\eta\left(Z^{\prime}\right)$ to the potential $\psi\left(Z^{\prime}\right)$ and the distribution functions $\hat{F}_{b}^{>}\left(W^{\prime}\right)$ and $\hat{F}_{b}^{<}\left(W^{\prime}\right)$.

To summarize, solutions to the nonlinear Vlasov equation $(75)$ that are time-stationary $\left(\partial / \partial T^{\prime}=0\right)$ in a frame of reference moving with normalized velocity $M=$ const are determined from (78), which in turn has exact solutions for the distribution function $\hat{F}_{b}\left(Z^{\prime}, V_{z}^{\prime}\right)$ of the general form given in Eq. (80), where $W^{\prime}$ is the total particle energy 


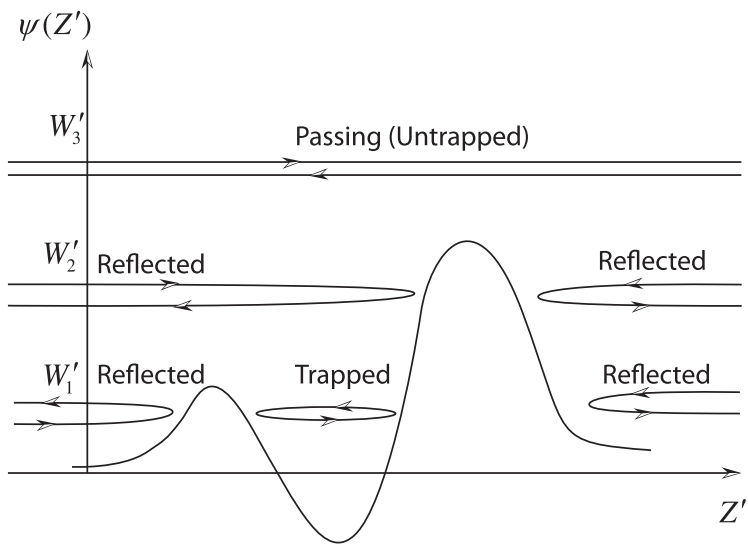

FIG. 11. Illustrative plot of the effective potential $\psi\left(Z^{\prime}\right)$ versus $Z^{\prime}$ occurring in Eq. (33) showing the three classes of particle orbits corresponding to: passing (untrapped) particles with energy $W_{3}^{\prime}$; reflected particles with energy $W_{2}^{\prime}$; and reflected or trapped particles (depending on the range of $Z^{\prime}$ ) with energy $W_{1}^{\prime}$. The form of $\psi\left(Z^{\prime}\right)$ in Fig. 11 corresponds to an isolated pulse with $\psi\left(Z^{\prime} \rightarrow \pm \infty\right)=0$.

(kinetic plus potential) defined in Eq. (79). Although the class of distributions described by Eqs. (79) and (80) is broad, it should be kept in mind that not all choices of distribution function $\hat{F}_{b}\left(W^{\prime}\right)$ are likely to be physically realized.

To reiterate the main features of the present model, for time-stationary solutions $\left(\partial / \partial T^{\prime}=0\right)$ in the moving frame, the normalized self-field potential $\psi\left(Z^{\prime}\right)$ and perturbed line density $\eta\left(Z^{\prime}\right)=\left[\lambda_{b}\left(Z^{\prime}\right)-\lambda_{b 0}\right] / \lambda_{b 0}$ are related by Eq. (76), and in turn $\eta\left(Z^{\prime}\right)$ is related self-consistently to the distribution function $\hat{F}_{b}\left(W^{\prime}\right)=\hat{F}_{b}^{>}\left(W^{\prime}\right)+\hat{F}_{b}^{<}\left(W^{\prime}\right)$ by Eq. (83), where $W^{\prime}=(1 / 2) V_{z}^{\prime 2}+\psi\left(Z^{\prime}\right)$ is the particle energy (kinetic plus potential). Therefore, for particles distributed in energy $W^{\prime}$ according to a specified functional

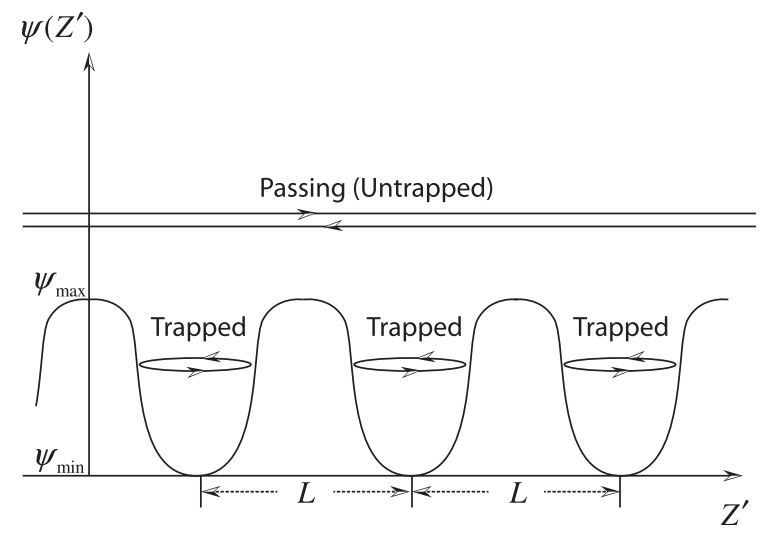

FIG. 12. Illustrative plot of the effective potential $\psi\left(Z^{\prime}\right)$ versus $Z^{\prime}$ for the case where $\psi\left(Z^{\prime}\right)$ has a nonlinear periodic waveform with $\psi\left(Z^{\prime}+L\right)=\psi\left(Z^{\prime}\right)$, where $L$ is the periodicity length. In the figure, passing particles with energy $W^{\prime}>\psi_{\max }$ are untrapped, whereas particles with energy $\psi_{\text {min }}<W^{\prime}<\psi_{\max }$ are trapped and exhibit periodic motion in the potential $\psi\left(Z^{\prime}\right)$. form for $\hat{F}_{b}\left(W^{\prime}\right)$, one can envision calculating selfconsistently $\eta\left[\psi\left(Z^{\prime}\right)\right]$ from Eq. (83), and $\psi\left(Z^{\prime}\right)$ from Eq. (76). Once $\psi\left(Z^{\prime}\right)$ is determined self-consistently for particles distributed in energy $W^{\prime}$ according to $\hat{F}_{b}\left(W^{\prime}\right)$, it is both reasonable and informative to examine the motion of an individual "test" particle with energy $W^{\prime}$ in the self-field potential $\psi\left(Z^{\prime}\right)$. For time-stationary potential $\psi\left(Z^{\prime}\right)$, particles move on constant energy surfaces with energy $W^{\prime}=$ $(1 / 2) V_{z}^{\prime 2}+\psi\left(Z^{\prime}\right)=$ const [see Eq. (79)]. For $W^{\prime}>\psi\left(Z^{\prime}\right)$, the orbit $Z^{\prime}\left(T^{\prime}\right)$ of a particle with energy $W^{\prime}$ is determined from $d Z^{\prime} / d T^{\prime}=V_{z}^{\prime}= \pm\left[2\left(W-\psi\left(Z^{\prime}\right)\right]^{1 / 2}\right.$. Keeping in mind that $\hat{F}_{b}\left(W^{\prime}\right)$ is the distribution of particles (in energy) that self-consistently generates the potential $\psi\left(Z^{\prime}\right)$, the preceding discussion forms the basis for examining qualitative features of the single-particle motion for different values of $W^{\prime}$, both for isolated pulse disturbances (Fig. 11), and nonlinear traveling wave distributions (Fig. 12).

Figure 11 shows an illustrative plot of the potential $\psi\left(Z^{\prime}\right)$ as a function of $Z^{\prime}$. Depending on the values of the energy $W^{\prime}$ and the range of $Z^{\prime}$, there are three classes of particle orbits: (i) particles that are reflected from the potential; (ii) particles that are trapped and undergo periodic motion; and (iii) passing (untrapped) particles that do not change direction, but pass over the potential maximum, first slowing down and then speeding up during the motion. For the trapped particles and the reflected particles, it follows that $\hat{F}^{>}\left(W^{\prime}\right)=\hat{F}^{<}\left(W^{\prime}\right)$ so that

$$
\hat{F}_{\operatorname{Tr}}\left(W^{\prime}\right)=\hat{F}_{\operatorname{Tr}}^{<}\left(W^{\prime}\right)+\hat{F}_{\operatorname{Tr}}^{>}\left(W^{\prime}\right)=2 \hat{F}_{\operatorname{Tr}}^{<}\left(W^{\prime}\right)=2 \hat{F}_{\operatorname{Tr}}^{>}\left(W^{\prime}\right)
$$

and

$$
\begin{aligned}
\hat{F}_{\text {Ref }}\left(W^{\prime}\right) & =\hat{F}_{\text {Ref }}^{<}\left(W^{\prime}\right)+\hat{F}_{\text {Ref }}^{>}\left(W^{\prime}\right)=2 \hat{F}_{\text {Ref }}^{<}\left(W^{\prime}\right) \\
& =2 \hat{F}_{\text {Ref }}^{>}\left(W^{\prime}\right) .
\end{aligned}
$$

On the other hand, for the passing (untrapped) particles, $\hat{F}_{\text {Un }}^{>}\left(W^{\prime}\right)$ and $\hat{F}_{\text {Un }}^{<}\left(W^{\prime}\right)$ can be specified independently, depending on whether the particles have $V_{z}^{\prime}>0$ or $V_{z}^{\prime}<0$, respectively.

The form of $\psi\left(Z^{\prime}\right)$ shown in Fig. 11 corresponds to a stationary isolated pulse in primed variables, with $\psi\left(Z^{\prime}= \pm \infty\right)=0$. By contrast, Fig. 12 shows a plot of $\psi\left(Z^{\prime}\right)$ versus $Z^{\prime}$ for the case where $\psi\left(Z^{\prime}\right)$ has a periodic nonlinear wave structure with

$$
\psi\left(Z^{\prime}+L\right)=\psi\left(Z^{\prime}\right) .
$$

From Fig. 12, trapped particles with energy $W^{\prime}$ in the range

$$
\psi_{\min }<W^{\prime}<\psi_{\max }
$$

exhibit periodic motion. On the other hand, passing particles with energy $W^{\prime}$ in the range (see Fig. 12) 


$$
W^{\prime}>\psi_{\max }
$$

correspond to untrapped particles that pass over the potential $\psi\left(Z^{\prime}\right)$, periodically speeding up and slowing down, but not changing their direction of motion. Furthermore, for the nonlinear periodic waveform for the potential $\psi\left(Z^{\prime}+L\right)=\psi\left(Z^{\prime}\right)$ shown in Fig. 12, it follows from Eqs. (76) and (83) that the waveform for the perturbation in line charge also satisfies $\eta\left(Z^{\prime}+L\right)=$ $\eta\left(Z^{\prime}\right)$. Here, $\eta\left(Z^{\prime}\right)$ is related to $\psi\left(Z^{\prime}\right)$ and the trappedparticle and untrapped-particle distribution functions by Eq. (83), which gives

$$
\begin{aligned}
1+\eta= & \int_{\psi}^{\psi_{\max }} d W^{\prime} \frac{\hat{F}_{\operatorname{Tr}}\left(W^{\prime}\right)}{\left[2\left(W^{\prime}-\psi\right)\right]^{1 / 2}} \\
& +\int_{\psi}^{\infty} d W^{\prime} \frac{\hat{F}_{U n}\left(W^{\prime}\right)}{\left[2\left(W^{\prime}-\psi\right)\right]^{1 / 2}}
\end{aligned}
$$

In Eq. (89), the integration over the trapped-particle distribution $\hat{F}_{\mathrm{Tr}}\left(W^{\prime}\right)$ is over the interval of $W^{\prime}$ corresponding to $\psi_{\min }<\psi<W^{\prime}<\psi_{\max }$, and the integration over the untrapped particle distribution $\hat{F}_{U n}\left(W^{\prime}\right)$ is over the interval of $W^{\prime}$ corresponding to $\psi_{\max }<\psi<W^{\prime}<\infty$.

Equations (76) and (89) can be used to determine detailed properties of self-consistent nonlinear periodic solutions for $\eta\left(Z^{\prime}\right)$ and $\psi\left(Z^{\prime}\right)$ for a broad range of choices of $\hat{F}_{\mathrm{Tr}}\left(W^{\prime}\right)$ and $\hat{F}_{\mathrm{Un}}\left(W^{\prime}\right)$. Furthermore, depending on system parameters, the amplitudes of the wave perturbations can range from small to moderately large amplitude. For purposes of illustration, the procedure for solving Eqs. (76) and (89) for the case of nonlinear periodic solutions for $\eta\left(Z^{\prime}\right)$ and $\psi\left(Z^{\prime}\right)$, we consider the special case where $\hat{F}_{\mathrm{Tr}}\left(W^{\prime}\right)=0$, and the untrapped distribution function has the monoenergetic form

$$
\hat{F}_{\mathrm{Un}}\left(W^{\prime}\right)=A \sqrt{2 W_{U}^{\prime}} \delta\left(W^{\prime}-W_{U}^{\prime}\right),
$$

where $W_{U}^{\prime}=$ const, $A=$ const, and $W_{U}^{\prime}>\psi_{\max }$ (see Fig. 12). Substituting $\hat{F}_{\mathrm{Tr}}\left(W^{\prime}\right)=0$ and Eq. (90) into Eq. (89) readily gives

$$
1+\eta\left(Z^{\prime}\right)=\frac{A}{\left[1-\psi\left(Z^{\prime}\right) / W_{U}^{\prime}\right]^{1 / 2}} .
$$

For present purpose, we choose the normalization constant $A$ in Eq. (91) such that the line density perturbation $\eta\left(Z^{\prime}\right)=$ $\lambda_{b}\left(Z^{\prime}\right) / \lambda_{b 0}-1$ and potential perturbation $\psi\left(Z^{\prime}\right)$ are simultaneously zero for all $Z^{\prime}$, i.e., $\eta\left(Z^{\prime}\right)=0$ for all $Z^{\prime}$, when $\psi\left(Z^{\prime}\right)=0$. From Eq. (91), this readily gives $A=1$ for the value of the constant $A$. Squaring Eq. (91) and solving for $\psi\left(Z^{\prime}\right)$ when $A=1$ readily gives

$$
\psi=W_{U}^{\prime}\left[1-\frac{1}{(1+\eta)^{2}}\right]
$$

Note that Eq. (92) determines $\psi\left(Z^{\prime}\right)$ as a function of $\eta\left(Z^{\prime}\right)$, which can be substituted into Eq. (76) to solve for $\eta\left(Z^{\prime}\right)$.

Similar to the analysis in Sec. III B for the class of nonlinear periodic traveling-wave solutions with $\eta\left(Z^{\prime}+L\right)=\eta\left(Z^{\prime}\right)$ and $\psi\left(Z^{\prime}+L\right)=\psi\left(Z^{\prime}\right)$, we examine Eqs. (76) and (92) for the case where the boundary conditions correspond to $\eta\left(Z^{\prime}=0\right)=0$ and $\left[\partial^{2} \eta / \partial Z^{\prime 2}\right]_{Z^{\prime}=0}=0$. Substituting Eq. (92) into Eq. (76) we readily obtain

$$
\frac{\partial^{2} \eta}{\partial Z^{\prime 2}}+\eta=W_{U}^{\prime}\left[1-\frac{1}{(1+\eta)^{2}}\right]
$$

which can also be expressed as

$$
\frac{\partial^{2} \eta}{\partial Z^{\prime 2}}+\frac{\partial V}{\partial \eta}=0
$$

where

$$
\begin{aligned}
\frac{\partial V}{\partial \eta} & =\eta-W_{U}^{\prime}\left[1-\frac{1}{(1+\eta)^{2}}\right] \\
& =\frac{\eta}{(1+\eta)^{2}}\left[\eta^{2}+\left(2-W_{U}^{\prime}\right) \eta+\left(1-2 W_{U}^{\prime}\right)\right]
\end{aligned}
$$

Note that Eq. (94) has the form of a dynamical equation of motion, with $\eta$ playing the role of displacement, $Z^{\prime}$ playing the role of time, and $V(\eta)$ playing the role of an effective potential, or so-called pseudopotential. Making use of Eq. (95), it is readily shown that

$$
\frac{\partial^{2} V}{\partial \eta^{2}}=1-\frac{2 W_{U}^{\prime}}{(1+\eta)^{3}}
$$

and

$$
\begin{aligned}
V(\eta) & =\frac{1}{2} \eta^{2}-W_{U}^{\prime}\left[\eta+\frac{1}{1+\eta}-1\right] \\
& =\frac{1}{2} \frac{\eta^{2}}{(1+\eta)^{2}}\left\{\eta+\left[1-2 W_{U}^{\prime}\right]\right\},
\end{aligned}
$$

where the constant of integration in Eq. (97) has been chosen so that $V(\eta=0)=0$.

Close examination of Eqs. (93)-(96) shows that Eq. (94) supports oscillatory solutions for $\eta\left(Z^{\prime}\right)$ about $\eta=0$ provided $\left[\partial^{2} V / \partial \eta^{2}\right]_{\eta=0}>0$, or equivalently,

$$
2 W_{U}^{\prime}<1
$$

When the inequality in Eq. (98) is satisfied, the plot of $V(\eta)$ versus $\eta$ has the characteristic shape illustrated in Fig. 13 for the choice of parameter $2 W_{U}^{\prime}<1$. Here, $V(\eta)$ has a minimum at $\eta=0$, and passes through zero at 


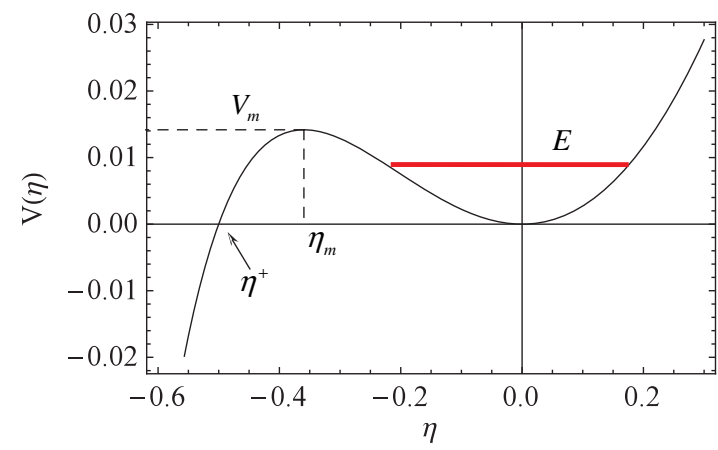

FIG. 13. Plot of $V(\eta)$ versus $\eta$ obtained from Eq. (97) for $2 W_{u}^{\prime}=0.5$ and $\eta^{+}=-0.5$. Here, $\eta_{m}=-0.36$, and $V\left(\eta_{m}\right)=0.014$. Nonlinear periodic solutions for $\eta\left(Z^{\prime}\right)$ exist for energy level $E$ in the range $0<E<V\left(\eta_{m}\right)$.

$$
\eta=\eta^{+}=-\left[1-2 W_{U}^{\prime}\right],
$$

where $V\left(\eta=\eta^{+}\right)=0$ [see Eqs. (97) and (99)]. Similar to the analysis in Sec. III B, Eq. (94) can be integrated to give the effective energy conservation relation $(1 / 2)\left[\partial \eta / \partial Z^{\prime}\right]^{2}+V(\eta)=E=$ const [see also Eq. (62)], where $E=(1 / 2)\left[\eta^{\prime}(0)\right]^{2}$ is the effective energy level. Referring to Fig. 13, Eqs. (94) and (97) support nonlinear periodic oscillatory solutions for $\eta\left(Z^{\prime}\right)$ for $E$ in the range $0<E<V_{m}$, where $V_{m} \equiv V\left(\eta=\eta_{m}\right)$ is the local maximum of $V(\eta)$ at $\eta=\eta_{m}$. For the choice of dimensionless parameter $2 W_{u}^{\prime}=1 / 2$ in Fig. 13, it is readily shown that $\eta_{m}=-0.360$ and $V_{m}=V\left(\eta=\eta_{m}\right)=0.014$.

Recall that the primed variables $\left(Z^{\prime}, V_{z}^{\prime}, T^{\prime}\right)$ are related to $\left(Z, V_{z}, T\right)$ by $Z^{\prime}=Z-M T, V_{z}^{\prime}=V_{z}-M$, and $T^{\prime}=T$, where $M=$ const is the dimensionless velocity of the traveling wave relative to the unprimed frame. Therefore, for a nonlinear wave that is time stationary $\left(\partial / \partial T^{\prime}=0\right)$ in the primed variables, it is reasonable to identify $W_{U}^{\prime}$ with $W_{U}^{\prime}=(1 / 2) M^{2}$ for a monoenergetic beam. In this case, we make the identification $2 W_{U}^{\prime}<1$, so the condition for Eqs. (94) and (95) to have nonlinear periodic solutions for $\eta\left(Z^{\prime}\right)$ [see Eq. (98)] can be expressed as

$$
M^{2}<1 .
$$

Typical numerical solutions for $\eta\left(Z^{\prime}\right)$, obtained by integrating Eq. (94) with $V(\eta)$ specified in Eq. (97), are illustrated in Figs. 14-17 for several choices of $M^{2}<1$ and different values of effective energy level $E$. These correspond to: $M^{2}=0.5, E=0.005, \eta_{m}=-0.360$, and $V\left(\eta_{m}\right)=0.014 \quad$ (Fig. 14); $M^{2}=0.5, \quad E=0.054883$, $\eta_{m}=-0.360$, and $V\left(\eta_{m}\right)=0.014$ (Fig. 15); $M^{2}=0.09$, $E=0.05, \eta_{m}=-0.764$, and $V\left(\eta_{m}\right)=0.181$ (Fig. 16); and $M^{2}=0.09, E=0.18, \eta_{m}=-0.764$, and $V\left(\eta_{m}\right)=0.181$ (Fig. 17). Figures 14-17 illustrate several interesting trends in the nonlinear periodic wave solutions for $\eta\left(Z^{\prime}\right)$. [These should be compared with the nonlinear periodic wave
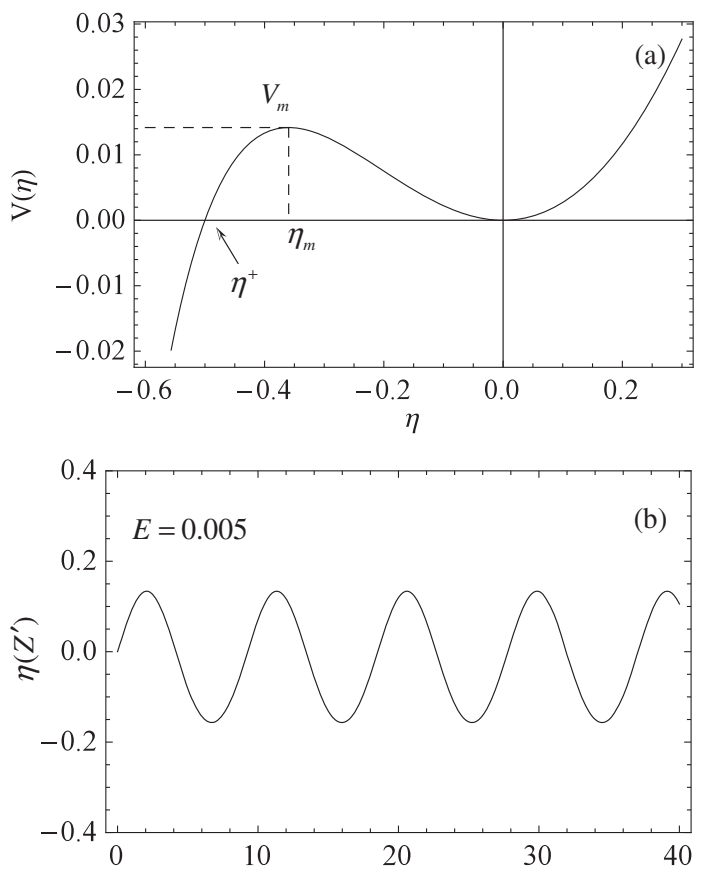

FIG. 14. Plots are shown for (a) $V(\eta)$ versus $\eta$, and (b) $\eta\left(Z^{\prime}\right)$ versus $Z^{\prime}$, obtained from Eq. (94) for $M^{2}=0.5, \eta^{\prime}(0)=0.1$, $E=(1 / 2)\left[\eta^{\prime}(0)\right]^{2}=0.005, \quad \eta^{+}=-0.5, \quad \eta_{m}=-0.36 \quad$ and $V\left(\eta_{m}\right)=0.014$.
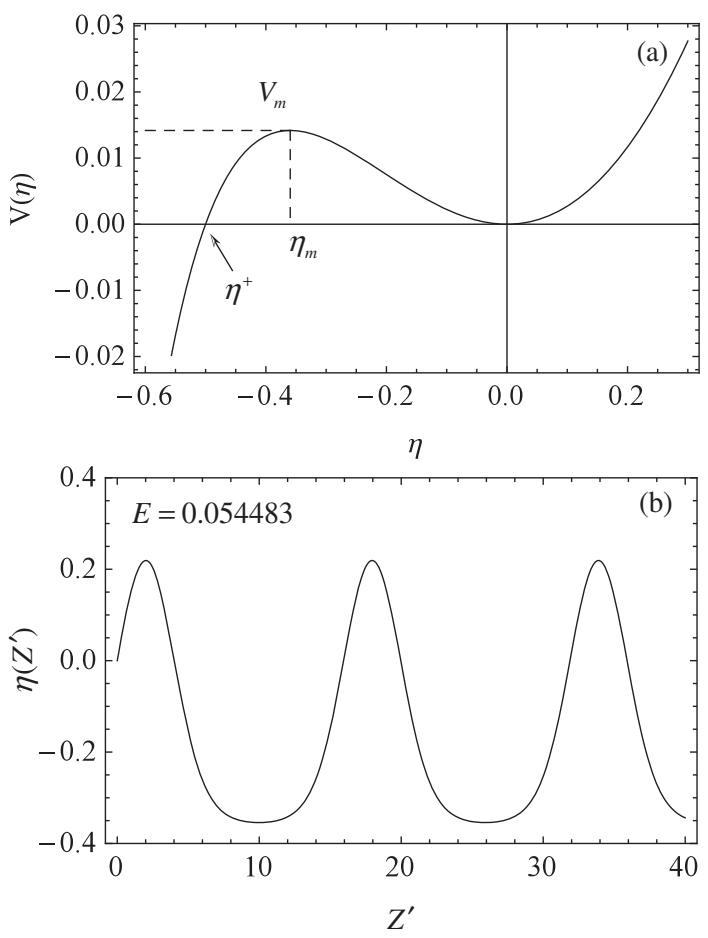

FIG. 15. Plots are shown for (a) $V(\eta)$ versus $\eta$, and (b) $\eta\left(Z^{\prime}\right)$ versus $Z^{\prime}$, obtained from Eq. (94) for $M^{2}=0.5, \eta^{\prime}(0)=0.1863$, $E=(1 / 2)\left[\eta^{\prime}(0)\right]^{2}=0.054883, \quad \eta^{+}=-0.5, \quad \eta_{m}=-0.36$ and $V\left(\eta_{m}\right)=0.014$. 


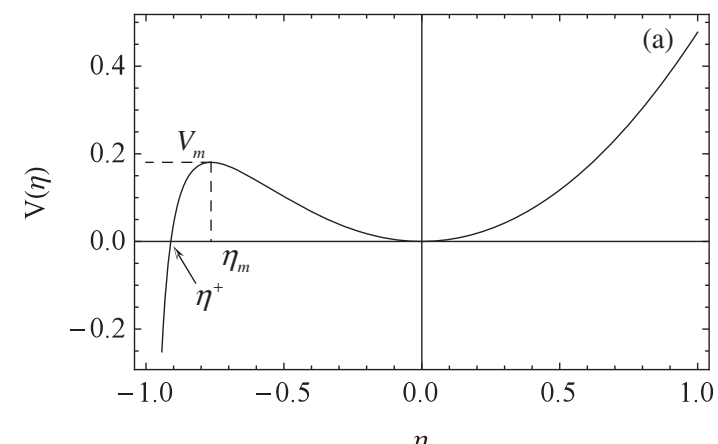

$\eta$

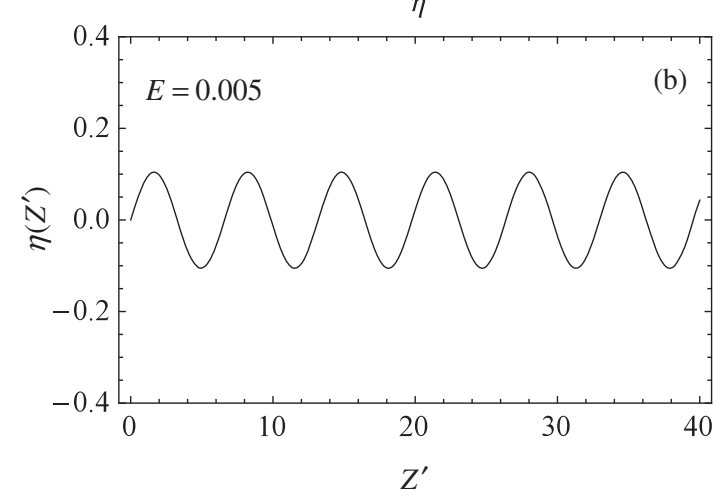

FIG. 16. Plots are shown for (a) $V(\eta)$ versus $\eta$, and (b) $\eta\left(Z^{\prime}\right)$ versus $Z^{\prime}$, obtained from Eq. (94) for $M^{2}=0.09, \eta^{\prime}(0)=0.1$, $E=(1 / 2)\left[\eta^{\prime}(0)\right]^{2}=0.05, \quad \eta^{+}=-0.91, \quad \eta_{m}=-0.764 \quad$ and $V\left(\eta_{m}\right)=0.181$.
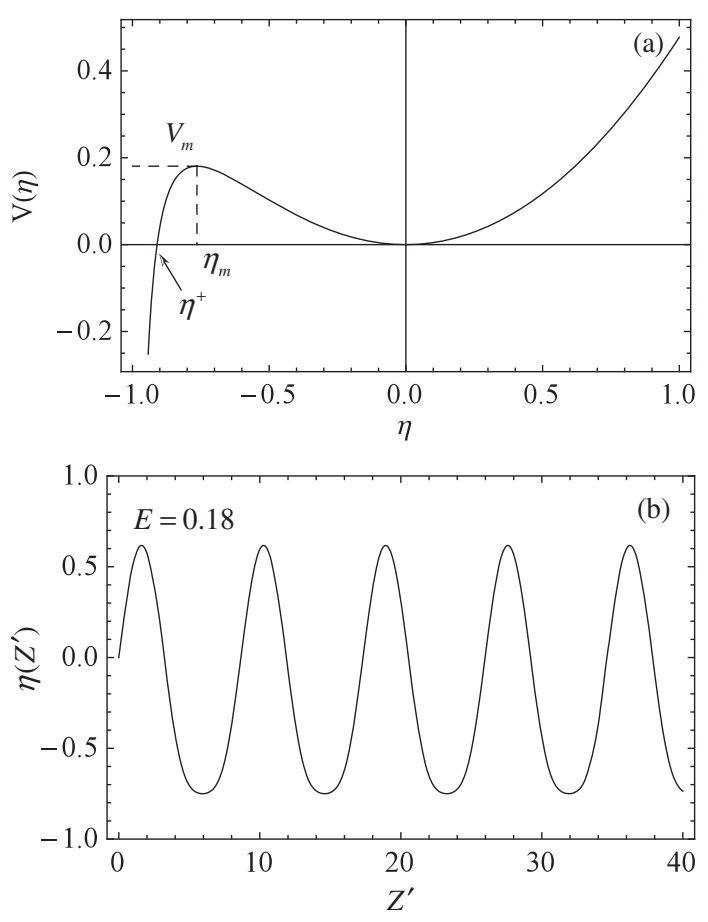

FIG. 17. Plots are shown for (a) $V(\eta)$ versus $\eta$, and (b) $\eta\left(Z^{\prime}\right)$ versus $Z^{\prime}$, obtained from Eq. (94) for $M^{2}=0.09, \eta^{\prime}(0)=0.6$, $E=(1 / 2)\left[\eta^{\prime}(0)\right]^{2}=0.18, \quad \eta^{+}=-0.91, \quad \eta_{m}=-0.764 \quad$ and $V\left(\eta_{m}\right)=0.181$. solutions in Figs. 2-5 obtained in Sec. III for the kinetic waterbag model.] First, for smaller values of $M^{2}$, the potential wells are deeper and broader (compare Figs. 14a and 15a with Figs. 16a and 17a). Furthermore, the nonlinear wave amplitudes tend to be large for sufficiently large values of energy level $E$ in the effective potential well (compare Figs. 15 and 17 with Figs. 14 and 16).

\section{CONCLUSIONS}

In this paper, the 1D kinetic model developed in Ref. [14] was used to describe the nonlinear longitudinal dynamics of intense beam propagation, allowing for moderate-to-large-amplitude modulation in the charge density of the beam particles. Particular emphasis has been placed on investigating detailed properties of nonlinear pulselike (soliton) and periodic traveling-wave disturbances propagating with constant normalized velocity $M=$ const relative to the beam frame. The 1D kinetic formalism [14] was briefly summarized in Sec. II A, and exact (local and nonlocal) nonlinear conservation constraints were derived in Sec. II B for the conserved particle number, momentum, and energy per unit length of the beam, making use of the nonlinear Vlasov equation for $F_{b}\left(z, p_{z}, t\right)$ in Eq. (1) and the expression for $\left\langle E_{z}\right\rangle(z, t)$ in Eq. (2). Removing the assumption of weak nonlinearity made in Ref. [15], Sec. III made use of the fully nonlinear kinetic waterbag model to investigate detailed properties of traveling nonlinear disturbances propagating with velocity $M=$ const relative to the beam frame. In normalized variables, $Z^{\prime}=Z-M T$ and $T^{\prime}=T$, the waveform of the disturbance was assumed to be time-stationary $\left(\partial / \partial T^{\prime}=0\right)$ in the frame moving with velocity $M=$ const. Nonlinear solutions were examined over a wide range of system parameters for both traveling-pulse (soliton) and nonlinear traveling-wave solutions in which the modulation in beam density was large-amplitude, corresponding to a strongly bunched beam. Finally, in Sec. IV we examined the kinetic model based on Eqs. (9) and (10) [equivalent to Eqs. (1) and (2)] for an even broader class of distribution functions $F_{b}\left(z, p_{z}, t\right)$. The analysis in Sec. IV parallels the original Bernstein-Greene-Kruskal (BGK) formulation of BGK solutions to the 1D VlasovPoisson equations [25,26], except for the fact that Eq. (10), which connects the effective potential $\langle\phi\rangle(z, t)$ to the line density $\lambda_{b}(z, t)$, has a very different structure than the $1 \mathrm{D}$ Poisson's equation used in the original BGK analysis. Depending on the choices of trapped-particle and untrapped-particle distribution functions, the kinetic model described in Sec. IV supports a broad range of nonlinear pulselike (soliton) solutions and periodic traveling-wave solutions that have stationary waveform in a frame of reference moving with velocity $M=$ const relative to the beam frame. Similar to Sec. III, the modulation in beam line density can have large amplitude, corresponding to a strong 
bunching of the beam particles. Specific examples were considered in Sec. IV corresponding to nonlinear periodic traveling-wave solutions of Eqs. (9) and (10).

In summary, the analysis in Secs. III and IV has identified a broad class of nonlinear traveling-wave and traveling-pulse (soliton) solutions with time-stationary waveform using a one-dimensional kinetic model based on Eqs. (1) and (2) [14]. Properties of these coherent nonlinear structures depend on the self-consistent interaction of the beam particles with the conducting wall through the geometric factors $g_{0}$ and $g_{2}$ occurring in Eqs. (2) and (3). Properties of the coherent structures also depend on the detailed distribution of particles in phase space $\left(z, p_{z}\right)$. For example, coherent traveling-wave structures can evolve as the nonlinear saturated state of mild or strong two-stream instability driven by a double-peaked distribution of beam particles in momentum space $p_{z}$. The linear (small-signal) theory for collective excitations based on Eqs. (1) and (2) has been developed in Sec. IV of Ref. [14], and will be explored in future studies as a basis for collective excitation of the class of coherent nonlinear structures described in the present article.

\section{ACKNOWLEDGMENTS}

This research was supported under the auspices of U.S. Department of Energy Contract No. DE-AC0209CH1 1466 with the Princeton Plasma Physics Laboratory.

[1] R. C. Davidson and H. Qin, Physics of Intense Charged Particle Beams in High Energy Accelerators (World Scientific, Singapore, 2001).

[2] M. Reiser, Theory and Design of Charged Particle Beams (Wiley, New York, 1994).

[3] A. W. Chao, Physics of Collective Beam Instabilities in High Energy Accelerators (Wiley, New York, 1993).
[4] D. A. Edwards and M. J. Syphers, An Introduction to the Physics of High Energy Accelerators, 1993 (Wiley, New York, 1993).

[5] J. D. Lawson, The Physics of Charged-Particle Beams (Oxford University Press, New York, 1988).

[6] T. P. Wangler, Principle of RF Linear Accelerators (John Wiley \& Sons, New York, 1998).

[7] I. Hofmann, Z. Naturforsch. Teil A 37A, 939 (1982).

[8] I. Hofmann, Laser Part. Beams 3, 1 (1985).

[9] R. Fedele, G. Miele, L. Palumbo, and V. Vaccaro, Phys. Lett. A 179, 407 (1993).

[10] L. K. Spentzouris, J. F. Ostiguy, and P. L. Colestock, Phys. Rev. Lett. 76, 620 (1996).

[11] O. Boine-Frankenheim, I. Hofmann, and G. Rumolo, Phys. Rev. Lett. 82, 3256 (1999).

[12] H. Schamel, Phys. Rev. Lett. 79, 2811 (1997).

[13] H. Schamel and R. Fedele, Phys. Plasmas 7, 3421 (2000).

[14] R. C. Davidson and E. A. Startsev, Phys. Rev. ST Accel. Beams 7, 024401 (2004).

[15] R. C. Davidson, Phys. Rev. ST Accel. Beams 7, 054402 (2004).

[16] R. C. Davidson, H. Qin, S. I. Tzenov, and E. A. Startsev, Phys. Rev. ST Accel. Beams 5, 084402 (2002).

[17] R. C. Davidson, Methods in Nonlinear Plasma Theory (Academic Press, New York, 1972).

[18] K. V. Roberts and H. L. Berk, Phys. Rev. Lett. 19, 297 (1967).

[19] F. Hohl and M. R. Feix, Astrophys. J. 147, 1164 (1967).

[20] D. Kordeweg and G. de Vries, Philos. Mag. Ser. 5 39, 422 (1895).

[21] H. Washimi and T. Taniuti, Phys. Rev. Lett. 17, 996 (1966).

[22] C. S. Gardner, J. M. Greene, M. D. Kruskal, and R. M. Miura, Phys. Rev. Lett. 19, 1095 (1967).

[23] E. Ott and R. Sudan, Phys. Fluids 12, 2388 (1969).

[24] R. C. Davidson, Methods in Nonlinear Plasma Theory (Ref. 17), p. 19.

[25] I. B. Bernstein, J. M. Greene, and M. D. Kruskal, Phys. Rev. 108, 546 (1957).

[26] R. C. Davidson, Methods in Nonlinear Plasma Theory (Ref. 17), p. 72. 